\title{
A positivity-preserving high-order semi-Lagrangian discontinuous Galerkin scheme for the Vlasov-Poisson equations
}

\author{
James A. Rossmanith ${ }^{\mathrm{a}, 1}$, David C. Seal ${ }^{\mathrm{a}}$ \\ ${ }^{a}$ Department of Mathematics, University of Wisconsin, 480 Lincoln Drive, Madison, WI
} 53706-1388, USA

\begin{abstract}
The Vlasov-Poisson equations describe the evolution of a collisionless plasma, represented through a probability density function (PDF) that self-interacts via an electrostatic force. One of the main difficulties in numerically solving this system is the severe time-step restriction that arises from parts of the PDF associated with moderate-to-large velocities. The dominant approach in the plasma physics community for removing these time-step restrictions is the so-called particle-in-cell (PIC) method, which discretizes the distribution function into a set of macro-particles, while the electric field is represented on a mesh. Several alternatives to this approach exist, including fully Lagrangian, fully Eulerian, and so-called semi-Lagrangian methods. The focus of this work is the semiLagrangian approach, which begins with a grid-based Eulerian representation of both the PDF and the electric field, then evolves the PDF via Lagrangian dynamics, and finally projects this evolved field back onto the original Eulerian mesh. In particular, we develop in this work a method that discretizes the $1+1$ Vlasov-Poisson system via a high-order discontinuous Galerkin (DG) method in phase space, and an operator split, semi-Lagrangian method in time. Secondorder accuracy in time is relatively easy to achieve via Strang operator splitting. With additional work, using higher-order splitting and a higher-order method of characteristics, we also demonstrate how to push this scheme to fourth-order accuracy in time. We show how to resolve all of the Lagrangian dynamics in such a way that mass is exactly conserved, positivity is maintained, and highorder accuracy is achieved. The Poisson equation is solved to high-order via the smallest stencil local discontinuous Galerkin (LDG) approach. We test the proposed scheme on several standard test cases.
\end{abstract}

Keywords: Discontinuous Galerkin; Semi-Lagrangian; Vlasov-Poisson; Plasma Physics; High-Order Schemes; Positivity-Preserving Limiters

\footnotetext{
Email addresses: rossmani@math.wisc.edu (James A. Rossmanith), seal@math.wisc.edu (David C. Seal)

${ }^{1}$ Corresponding author
} 


\section{Introduction}

The Vlasov equation in its various incarnations (e.g., Vlasov-Maxwell, VlasovDarwin, and Vlasov-Poisson) models the dynamics of collisionless plasma. Plasma is the state of matter where electrons have dissociated from their nuclei, creating a mixture of interacting charged particles. This mixture can evolve via a variety of effects, including electromagnetic interactions and through particle-particle collisions. In the collionless limit, the mean free-path is much larger than the characteristic length scale of the plasma; and therefore, particle-particle collisions are dropped from the mathematical model. Vlasov models are widely used in both astrophysical applications (e.g., 6, 8, 37]), as well as in laboratory settings (e.g., [11, 40, 33, 10, 28]).

The development of accurate and efficient numerical methods for the solution of the Vlasov equations are faced with a variety of numerical challenges, the most important of which we describe below.

- High dimensionality. The Vlasov system is a nonlinear and nonlocal advection equation in six phase space dimensions $\left(\mathbf{x} \in \mathbb{R}^{3}\right.$ and $\left.\mathbf{v} \in \mathbb{R}^{3}\right)$ and time - this of often referred to as $3+3+1$ dimensions. Even though the Vlasov equation is in many ways mathematically simpler than fluid models, the fact that it lives in a space of twice the number of dimensions makes it computationally much more expensive to solve.

- Conservation and positivity. In fluid models, conservation of mass, momentum, and energy are often relatively easy to guarantee in a numerical discretization, since each of these quantities is a dependent variable of the system. In Vlasov models it is generally more difficult to exactly maintain these quantities in the numerical discretization. Exact positivity of the probability density function is also not guaranteed by many standard discretizations of the Vlasov system; and therefore, additional work in choosing the correct approximation spaces is often required.

- Small time steps due to $\mathbf{v} \in \mathbb{R}^{3}$. In the non-relativistic case, the advection velocity of the density function in phase space depends linearly on the components of the velocity vector $\mathbf{v} \in \mathbb{R}^{3}$ (see equation (5) in $\$ 2$ ). Since it is in general possible to have "particles" in the Vlasov system that travel arbitrarily fast, there will be a severe time-step restriction, relative to the dynamics of interest, that arises from parts of the PDF associated with moderate-to-large velocities.

Several approaches have been introduced to try and solve some of these problems, including particle-in-cell methods, Lagrangian particle methods, and grid-based semi-Lagrangian methods. We briefly summarize each of these approaches below.

- Particle-in-cell methods. Particle-in-cell (PIC) methods are ubiquitous in both astrophysical (e.g., [40]) and laboratory plasma (e.g., 8] ) application problems. The basic approach is outlined in the celebrated textbooks 
of Birdsall and Langdon [7] and Hockney and Eastwood [27, both of which appeared in the mid-to-late 1980s. Modern improvements to these methods are still topics of current research (e.g., adaptive mesh refinement 40], very high-order variants [29, 30, etc...). The basic idea is that the distribution function is discretized into a set of macro-particles (Lagrangian representation), while the electromagnetic field is represented on a mesh (Eulerian representation). The main advantage of this approach is that positivity and mass conservation are essentially automatic, the small time step restriction is removed due to the fact that the particles are evolved in a Lagrangian framework, and the electromagnetic equations can be solved via standard mesh-based methods. The main disadvantages of this method are: (1) numerical errors are introduced due to the interpolations that must de done to exchange information between the particles and fields, and (2) error control is non-trivial since particles may either cluster or generate rarefied regions during the evolution of the plasma.

- Lagrangian particle methods. One possible alternative to the PIC methodology is to go to a completely Lagrangian framework - this removes the need to interpolate between the particles and fields. Such approaches are commonplace in several application areas such as many body dynamics in astrophysics [3, vortex dynamics [31, as well as in plasma physics [12. The key is that the potential (e.g., gravitational potential, streamfunction, or electric potential) is calculated by integrating the point charges represented by the Lagrangian particles against a Green's function. Since the charges are point particles, evaluating this integral reduces to computing sums over the particles. Naive methods would need $\mathcal{O}\left(N^{2}\right)$ floating point operations to evaluate all of these sums, where $N$ is the number of particles, but fast summation methods such as treecode methods [3, 31] and the fast multipole method [24] can be used to reduce this to $\mathcal{O}(N \log N)$. The main disadvantage of this approach is that it relies on having a Green's function, which for more complicated dynamics (i.e., full electromagnetism), may be difficult to obtain.

- Semi-Lagrangian grid-based methods. Another alternative to PIC is to switch to a completely grid-based method. Such an approach allows for a variety of high-order spatial discretizations, and can be evolved forward in time via so-called semi-Lagrangian time-stepping. The basic idea is that the PDF sits initially on a grid; the PDF is then evolved forward in time using Lagrangian dynamics; and finally, the new PDF is projected back onto the original mesh. This gives many of the advantages of particle methods (i.e., no small time-step restrictions), but retains a nice grid structure for both the PDF and the fields, allowing extension to very highorder accuracy. There have been several contributions to this approach over the last few years. One of the first papers that developed a viable semi-Lagrangian method was put forward by Cheng and Knorr 9. More recent activity on this approach includes the work of Parker and Hitchon 
33], Sonnendrücker and his collaborators (see for example [20, 22, 38, 18,

5, 19, 21, 4]), and Christlieb and Qiu [34.

The goal of the current work is to develop a high-order, grid-based, semiLagrangian method for solving the $1+1$ Vlasov-Poisson equation. We focus on the $1+1$ case, leaving the problem of high-dimensionality for future work. Our discretization is based on high-order discontinuous Galerkin representations and a high-order operator split semi-Lagrangian time-stepping method. We argue that this approach is a promising method that produces very accurate results at relatively low computational expense.

This paper begins with a brief review of the Vlasov equations in $\$ 2$ Part of the focus of the present work is to develop a higher-order version of the classical Cheng and Knorr [9] operator splitting method, which we review in 33 . The spatial discretization for the proposed method will be based on the discontinuous Galerkin method, which we briefly review in $\$ 4$. The heart of this paper is $\$ 5$. which details all the aspects of the proposed method, and in particular, explains how to achieve high-order in space and time, mass conservation, and positivity of the distribution function. Finally in 6 we apply the proposed scheme to a variety of standard test cases for the Vlasov-Poisson system, including the two-stream instability problem and Landau damping.

\section{Mathematical equations}

The Vlasov system describes the evolution of a probability density function $(\mathrm{PDF})$ in phase space:

$$
f_{s}(t, \mathbf{x}, \mathbf{v}): \mathbb{R}^{+} \times \mathbb{R}^{d} \times \mathbb{R}^{d} \rightarrow \mathbb{R}^{S},
$$

where $d$ is the spatial dimension and $S$ represents the number of plasma species. This PDF denotes the probability of finding a particle of species $s$ at time $t$, at location $\mathbf{x}$, and with velocity $\mathbf{v}$. Although the PDF is not itself a physical observable, its moments represent various physically observable quantities:

$$
\begin{aligned}
\rho_{s}(t, \mathbf{x}) & :=\int_{\mathbb{R}^{d}} f_{s} d \mathbf{v}, & & \text { (mass density of species } s), \\
\rho_{s} \mathbf{u}_{s}(t, \mathbf{x}) & :=\int_{\mathbb{R}^{d}} \mathbf{v} f_{s} d \mathbf{v}, & & \text { (momentum density of species } s), \\
\mathcal{E}_{s}(t, \mathbf{x}) & :=\frac{1}{2} \int_{\mathbb{R}^{d}}\|\mathbf{v}\|^{2} f_{s} d \mathbf{v}, & & \text { (energy density of species } s) .
\end{aligned}
$$

Under the assumptions of a non-relativistic and collisionless plasma, the PDF for each species obeys the Vlasov equation, which is an advection equation in $(\mathbf{x}, \mathbf{v})$ phase space:

$$
\frac{\partial f_{s}}{\partial t}+\mathbf{v} \cdot \nabla_{\mathbf{x}} f_{s}+\frac{q_{s}}{m_{s}}(\mathbf{E}+\mathbf{v} \times \mathbf{B}) \cdot \nabla_{\mathbf{v}} f_{s}=0 .
$$


The "particles" represented by this kinetic description do not interact through collisional processes; and instead, are only coupled indirectly through the electromagnetic field. In general, the electromagnetic field satisfies Maxwell's equations:

$$
\begin{aligned}
\frac{\partial}{\partial t}\left[\begin{array}{l}
\mathbf{B} \\
\mathbf{E}
\end{array}\right]+\nabla \times\left[\begin{array}{c}
\mathbf{E} \\
-c^{2} \mathbf{B}
\end{array}\right] & =\left[\begin{array}{c}
0 \\
-c^{2} \mathbf{J}
\end{array}\right], \\
\nabla \cdot \mathbf{B}=0, \quad \nabla \cdot \mathbf{E} & =c^{2} \sigma,
\end{aligned}
$$

where $\mathbf{B}$ is the magnetic field, $\mathbf{E}$ is the electric field, and the total charge density and total current densities are given by the following:

$$
\sigma=\sum_{s} \frac{q_{s}}{m_{s}} \rho_{s}, \quad \mathbf{J}=\sum_{s} \frac{q_{s}}{m_{s}} \rho_{s} \mathbf{u}_{s} .
$$

Note that the electromagnetic field variables, $\mathbf{B}(t, \mathbf{x})$ and $\mathbf{E}(t, \mathbf{x})$, as well as the mass and momentum densities, $\rho_{s}(t, \mathbf{x})$ and $\rho_{s} \mathbf{u}_{s}(t, \mathbf{x})$, only depend on time and the spatial coordinates $\mathbf{x}$.

In this work we will not consider the full Vlasov-Maxwell system for a many species plasma; and instead, we only consider the single-species Vlasov-Poisson equation. To arrive at the Vlasov-Poisson system, we start with Vlasov-Maxwell and assume that the charges are slow-moving in comparison to the speed of light; this allows us to replace the full electromagnetic equations with electrostatics. Furthermore, we consider only two-species: one dynamically evolving species, which we take without loss of generality to have positive charge and unit mass $m=1$, and one stationary background species that has a charge of opposite sign to the dynamic species. Because the background charge is stationary, we will only need to solve a single-species Vlasov equation. These assumptions conspire to form the Vlasov-Poisson equations:

$$
\begin{gathered}
f_{, t}+\mathbf{v} \cdot f_{, \mathbf{x}}+\nabla \phi \cdot f_{, \mathbf{v}}=0, \\
\nabla^{2} \phi=\rho(t, \mathbf{x})-\rho_{0},
\end{gathered}
$$

where $\phi$ is the electric potential: $\nabla \phi=\mathbf{E}$, and $-\rho_{0}$ is the stationary background charge density.

The Vlasov-Poisson system contains an infinite number of quantities that are conserved in time. These can be used as diagnostics in a numerical discretization. We list four quantities that will be used in diagnosing our proposed scheme, all of which should remain constant in time:

$$
\begin{aligned}
\|f\|_{L_{1}} & :=\int_{\mathbb{R}^{d}} \int_{\mathbb{R}^{d}}|f| d \mathbf{v} d \mathbf{x}, \\
\|f\|_{L_{2}} & :=\left(\int_{\mathbb{R}^{d}} \int_{\mathbb{R}^{d}} f^{2} d \mathbf{v} d \mathbf{x}\right)^{\frac{1}{2}}, \\
\text { Total energy } & :=\frac{1}{2} \int_{\mathbb{R}^{d}} \int_{\mathbb{R}^{d}}\|\mathbf{v}\|^{2} f d \mathbf{v} d \mathbf{x}+\frac{1}{2} \int_{\mathbb{R}^{d}}\|\mathbf{E}\|^{2} d \mathbf{x}, \\
\text { Entropy } & :=-\int_{\mathbb{R}^{d}} \int_{\mathbb{R}^{d}} f \log (f) d \mathbf{v} d \mathbf{x} .
\end{aligned}
$$


Finally, we point out that in the current work we are concerned exclusively with the $1+1$ dimensional version of the above equations with periodic boundary conditions in $x$. In this case, the Vlasov-Poisson system on $\Omega=(t, x, v) \in$ $\mathbb{R}^{+} \times[-L, L] \times \mathbb{R}$ is:

$$
\begin{gathered}
f_{, t}+v f_{, x}+E(t, x) f_{, v}=0, \\
E_{, x}=\rho(t, x)-\rho_{0},
\end{gathered}
$$

with periodic boundary conditions:

$$
f(t,-L, v)=f(t, L, v), \quad E(t,-L)=E(t, L), \quad \text { and } \quad \phi(t,-L)=\phi(t, L) .
$$

In these expressions we used the shorthand notation:

$$
x:=x^{1}, \quad v:=v^{1}, \quad \text { and } \quad E:=E^{1} .
$$

The total and background densities are

$$
\rho(t, x):=\int_{-\infty}^{\infty} f(t, x, v) d v \quad \text { and } \quad \rho_{0}:=\frac{1}{2 L} \int_{-L}^{L} \rho(t, x) d x .
$$

Note that $\rho_{0}$ is in fact constant in time, due to conservation of mass on the periodic domain $[-L, L]$.

\section{Strang operator splitting}

If we momentarily freeze the electric field in time, the Vlasov equation (9) can be viewed as an advection equation of the following form:

$$
f_{, t}+\mathbf{a}(\mathbf{v}) \cdot f_{, \mathbf{x}}+\mathbf{b}(\mathbf{x}) \cdot f_{, \mathbf{v}}=0 .
$$

Cheng and Knorr [9] realized that such an equation can be handled very efficiently if split into the following two sub-problems:

$$
\begin{array}{ll}
\text { Problem } \mathcal{A}: & f_{, t}+\mathbf{a}(\mathbf{v}) \cdot f_{, \mathbf{x}}=0 \\
\text { Problem } \mathcal{B}: & f_{, t}+\mathbf{b}(\mathbf{x}) \cdot f_{, \mathbf{v}}=0 .
\end{array}
$$

The key benefit of this splitting is that each operator is now a constant coefficient advection equation (i.e., the transverse coordinate acts only as a parameter), each of which can be handled very simply with a variety of spatial discretization and semi-Lagrangian time-stepping. The down side of this approach, of course, is the introduction of splitting error.

Cheng and Knorr 9 developed a second order accurate version of this scheme via Strang operator splitting 39. Their scheme is summarized in Algorithm 1 It is worth pointing out that the electric field computed in Step 2, $\mathbf{E}^{n+\frac{1}{2}}$, is second order accurate in time, even though it is computed after advection in the $\mathbf{x}$ variables only. 
Claim. Assuming that the current solution at time $t=t^{n}$ is known exactly, and that each step in Algorithm 1 is carried out exactly in space, velocity, and time, the density computed in Step 2 is second order accurate in time:

$$
\rho^{n+\frac{1}{2}}=\rho\left(t^{n}+\frac{\Delta t}{2}, \mathbf{x}\right)+\mathcal{O}\left(\Delta t^{2}\right) .
$$

This also implies that the electric field in Step 2 is second order accurate in time:

$$
\mathbf{E}^{n+\frac{1}{2}}=\mathbf{E}\left(t^{n}+\frac{\Delta t}{2}, \mathbf{x}\right)+\mathcal{O}\left(\Delta t^{2}\right) .
$$

Proof. By assumption the PDF after the first step satisfies the following relationship:

$$
\tilde{f}(\mathbf{x}, \mathbf{v}):=f\left(t^{n}, \mathbf{x}-\frac{\Delta t}{2} \mathbf{v}, \mathbf{v}\right) .
$$

We integrate this relationship in velocity to compute the density at time $t^{n}+\frac{\Delta t}{2}$ :

$$
\begin{aligned}
\rho^{n+\frac{1}{2}} & :=\int_{\mathbf{v}} \tilde{f}(\mathbf{x}, \mathbf{v}) d \mathbf{v}=\int_{\mathbf{v}} f\left(t^{n}, \mathbf{x}-\frac{\Delta t}{2} \mathbf{v}, \mathbf{v}\right) d \mathbf{v} \\
& =\int_{\mathbf{v}} f\left(t^{n}, \mathbf{x}, \mathbf{v}\right) d \mathbf{v}-\frac{\Delta t}{2} \nabla_{\mathbf{x}} \cdot\left\{\int_{\mathbf{v}} \mathbf{v} f\left(t^{n}, \mathbf{x}, \mathbf{v}\right) d \mathbf{v}\right\}+\mathcal{O}\left(\Delta t^{2}\right) \\
& =\rho^{n}-\frac{\Delta t}{2} \nabla_{\mathbf{x}} \cdot\left(\rho^{n} \mathbf{u}^{n}\right)+\mathcal{O}\left(\Delta t^{2}\right) .
\end{aligned}
$$

Finally, we use the fact that

$$
\rho_{, t}^{n}=-\nabla_{\mathbf{x}}\left(\rho^{n} \mathbf{u}^{n}\right)
$$

in order to assert that

$$
\rho^{n+\frac{1}{2}}=\rho^{n}+\frac{\Delta t}{2} \rho_{, t}^{n}+\mathcal{O}\left(\Delta t^{2}\right)=\rho\left(t+\frac{\Delta t}{2}, \mathbf{x}\right)+\mathcal{O}\left(\Delta t^{2}\right),
$$

which proves the claim.

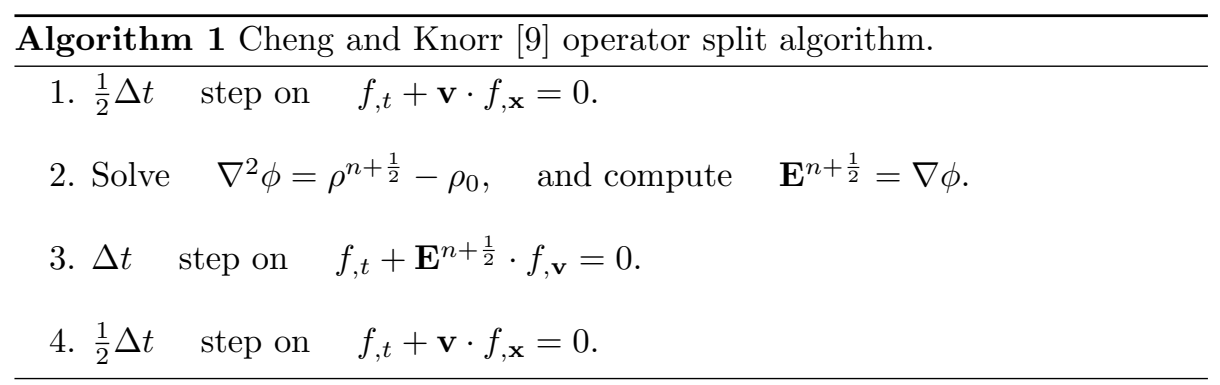


The original method of Cheng and Knorr [9] employs a cubic spline spatial discretization. In the past few years, work on semi-Lagrangian solvers using Algorithm 1 with a variety of spatial discretizations, including modified cubic spline interpolants, has been carried out by Sonnendrücker and his collaborators (see for example [20, 22, 38, 18, 5, 19, 21, 4]). Another recent contribution to this approach was the method of Christlieb and Qiu [34, who combined the operator split method with high-order WENO (weighted essentially non-oscillatory) finite differences for the spatial derivatives.

The focus of the present work is to again consider operator splitting techniques, but this time with high-order discontinuous Galerkin (DG) method for the spatial discretization, and with fourth-order operator splitting techniques. We describe the basic DG framework in the next section $\$ 4$ and various details of both a second and a fourth-order in time operator split approach in $\$ 5$.

\section{The discontinuous Galerkin (DG) method}

The modern form of the discontinuous Galerkin (DG) method was developed in a series of papers by Bernardo Cockburn, Chi-Wang Shu, and their collaborators [16, 15, 14, 13, 17. In this section we briefly review the DG method for a general two-dimensional conservation law on a Cartesian mesh. This section will also serve to introduce the notation that we will use throughout this paper.

Consider a general 2D conservation law of the form:

$$
q_{, t}+f(q, t, \mathbf{x})_{, x}+g(q, t, \mathbf{x})_{, y}=0, \quad \text { in } \quad \mathbf{x} \in \Omega \subset \mathbb{R}^{2},
$$

with appropriate initial and boundary conditions. In this equation $q(t, \mathbf{x}) \in \mathbb{R}^{m}$ is the vector of conserved variables and $f(q, t, \mathbf{x}), g(q, t, \mathbf{x}) \in \mathbb{R}^{m}$ are the flux functions in the $x$ and $y$-directions, respectively. We assume that equation (19) is hyperbolic, meaning that the family of $m \times m$ matrices defined by

$$
A(q, \mathbf{x} ; \mathbf{n})=\mathbf{n} \cdot\left(\frac{\partial f}{\partial q}, \frac{\partial g}{\partial q}\right)^{T}
$$

are diagonalizable with real eigenvalues for all $\mathbf{x}$ and $q$ in the domain of interest and for all $\|\mathbf{n}\|=1$.

We construct a Cartesian grid over $\Omega=\left[a_{x}, b_{x}\right] \times\left[a_{y}, b_{y}\right]$, with uniform grid spacing $\Delta x$ and $\Delta y$ in each coordinate direction. The mesh elements are centered at the coordinates

$$
x_{i}=a_{x}+\left(i-\frac{1}{2}\right) \Delta x \quad \text { and } \quad y_{j}=a_{y}+\left(j-\frac{1}{2}\right) \Delta y .
$$

On this grid we define the broken finite element space

$$
W^{h}=\left\{w^{h} \in L_{\infty}(\Omega):\left.w^{h}\right|_{\mathcal{T}} \in P^{q}, \forall \mathcal{T} \in \mathcal{T}_{h}\right\},
$$

where $W^{h}$ is shorthand notation for $W^{\Delta x, \Delta y}$. The above expression means that on each element $\mathcal{T}, w^{h}$ will be a polynomial of degree at most $q$, and no 
continuity is assumed across element edges. Each element can be mapped to the canonical element $(\xi, \eta) \in[-1,1] \times[-1,1]$ via the linear transformation:

$$
x=x_{i}+\xi \frac{\Delta x}{2}, \quad y=y_{j}+\eta \frac{\Delta y}{2} .
$$

The normalized Legendre polynomials up to degree four on the canonical element can be written as

$$
\begin{aligned}
\varphi^{(\ell)}=\{ & 1, \sqrt{3} \xi, \sqrt{3} \eta, 3 \xi \eta, \frac{\sqrt{5}}{2}\left(3 \xi^{2}-1\right), \frac{\sqrt{5}}{2}\left(3 \eta^{2}-1\right), \\
& \frac{\sqrt{15}}{2} \eta\left(3 \xi^{2}-1\right), \frac{\sqrt{15}}{2} \xi\left(3 \eta^{2}-1\right), \frac{\sqrt{7}}{2}\left(5 \xi^{3}-3 \xi\right), \frac{\sqrt{7}}{2}\left(5 \eta^{3}-3 \eta\right), \\
& \frac{\sqrt{21}}{2} \eta\left(5 \xi^{3}-3 \xi\right), \frac{\sqrt{21}}{2} \xi\left(5 \eta^{3}-3 \eta\right), \frac{5}{4}\left(3 \xi^{2}-1\right)\left(3 \eta^{2}-1\right), \\
& \left.\frac{105}{8} \xi^{4}-\frac{45}{4} \xi^{2}+\frac{9}{8}, \frac{105}{8} \eta^{4}-\frac{45}{4} \eta^{2}+\frac{9}{8}\right\}
\end{aligned}
$$

These basis functions are orthonormal with respect to the following inner product:

$$
\left\langle\varphi^{(m)}, \varphi^{(n)}\right\rangle:=\frac{1}{4} \int_{-1}^{1} \int_{-1}^{1} \varphi^{(m)}(\xi, \eta) \varphi^{(n)}(\xi, \eta) d \xi d \eta=\delta_{m n} .
$$

We will look for approximate solutions of 19 that have the following form:

$$
\left.q^{h}(t, \xi, \eta)\right|_{\mathcal{T}_{i j}}:=\sum_{k=1}^{M(M+1) / 2} Q_{i j}^{(k)}(t) \varphi^{(k)}(\xi, \eta),
$$

where $M$ is the desired order of accuracy in space (i.e., for fifth order: $M=5$ and $M(M+1) / 2=15)$. The Legendre coefficients of the initial conditions at $t=0$ are determined from the $L_{2}$-projection of $q^{h}(x, y, 0)$ onto the Legendre basis functions:

$$
Q_{i j}^{(k)}(0):=\left\langle q^{h}(0, \xi, \eta), \varphi^{(k)}(\xi, \eta)\right\rangle .
$$

In practice, these double integrals are evaluated using standard 2D Gaussian quadrature rules involving $M^{2}$ points.

In order to determine the Legendre coeficients for $t>0$, we multiply conservation law 19 by the test function $\varphi^{(\ell)}$ and integrate over the grid cell $\mathcal{T}_{i j}$. After the appropriate integrations-by-part, we arrive at the following semidiscrete evolution equations:

$$
\frac{d}{d t} Q_{i j}^{(\ell)}=\mathcal{L}_{i j}^{(\ell)}(Q, t):=N_{i j}^{(\ell)}-\frac{\Delta \mathcal{F}_{i j}^{(\ell)}}{\Delta x}-\frac{\Delta \mathcal{G}_{i j}^{(\ell)}}{\Delta y},
$$


where

$$
\begin{aligned}
N_{i j}^{(\ell)} & =\frac{1}{2} \int_{-1}^{1} \int_{-1}^{1}\left[\frac{1}{\Delta x} \varphi_{, \xi}^{(\ell)} f\left(q^{h}, t, \mathbf{x}\right)+\frac{1}{\Delta y} \varphi_{, \eta}^{(\ell)} g\left(q^{h}, t, \mathbf{x}\right)\right] d \xi d \eta, \\
\Delta \mathcal{F}_{i j}^{(\ell)} & =\left[\frac{1}{2} \int_{-1}^{1} \varphi^{(\ell)} f\left(q^{h}, t, \mathbf{x}\right) d \eta\right]_{\xi=-1}^{\xi=1}, \\
\Delta \mathcal{G}_{i j}^{(\ell)} & =\left[\frac{1}{2} \int_{-1}^{1} \varphi^{(\ell)} g\left(q^{h}, t, \mathbf{x}\right) d \xi\right]_{\eta=-1}^{\eta=1} .
\end{aligned}
$$

The integrals in 28 can be numerically approximated via standard 2D Gaussian quadrature rules involving $(M-1)^{2}$ points. The integrals in 29$)$ and 30 can be approximated with standard 1D Gauss quadrature rules involving $M$ points.

\section{A high-order semi-Lagrangian DG method}

We describe in this section a semi-Lagrangian discontinuous Galerkin method for solving the Vlasov-Poisson system. This method will have all of the following properties:

1. Unconditionally stable;

2. High-order accurate in space ( $5^{\text {th }}$ order);

3. High-order accurate in time ( $4^{\text {th }}$ order);

4. Mass conservative; and

5. Positivity-preserving.

All of these properties are explained in detail in this section.

We begin by explaining the basic idea on the constant coefficient $1 \mathrm{D}$ advection equation in $\$ 5.1$ The extension of this $1 \mathrm{D}$ scheme to the $1+1$ Vlasov equation via Strang operator splitting is described in section $\$ 5.2$ A simple and efficient local discontinuous Galerkin solver for the Poisson equation is described in $\$ 5.3$. The generalization of the Strang-split scheme to higher-order splitting is shown in $\$ 5.4$. Basic properties including conservation of mass and positivity in the mean are proved in $\$ 5.5$. Finally, in $\$ 5.6$ a limiter that provides global pointwise positivity is described.

\subsection{A toy problem: the $1 D$ advection equation}

Consider the 1D constant coefficient advection equation:

$$
f_{, t}+v f_{, x}=0, \quad(t, x) \in \mathbb{R}^{+} \times \mathbb{R},
$$

with initial condition $f(0, x)$. For simplicity of exposition, let's assume in the discussion below that that $v>0$; the extension to the case $v<0$ is straightforward. We consider solving this equation on a uniform mesh of elements, $\mathcal{T}_{i}$, that each have width $\Delta x$. We begin by projecting the initial condition onto the mesh:

$$
\left.F^{h}(0, \xi)\right|_{\mathcal{T}_{i}}=\sum_{\ell=1}^{M} F_{i}^{(\ell)}(0) \varphi_{1 \mathrm{D}}^{(\ell)}(\xi),
$$


(a)

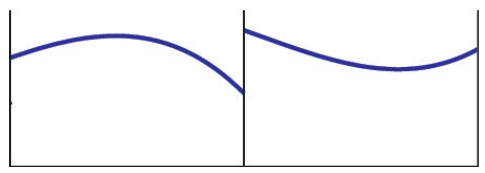

(b)

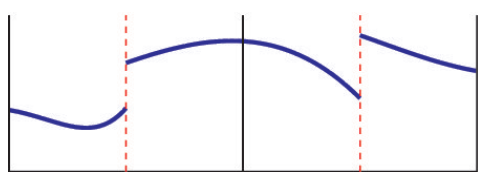

(c)

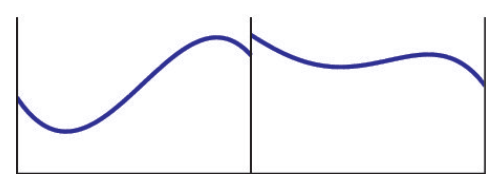

Figure 1: Illustration of the shift + project method for solving the constant coefficient advection equation in 1D as described in 55.1 Panel (a) shows piecewise polynomial initial data; Panel (b) shows the initial data shifted by some amount (i.e., the exact evolution of the initial data); and finally, Panel (c) shows the solution after it has been re-projected back onto the original piecewise polynomial basis.

where $F^{h}$ represents the finite dimensional approximation of $f(t, x), M$ is the desired order of accuracy, and $\varphi_{1 \mathrm{D}}^{(\ell)}(\xi)$ are the 1D Legendre basis functions:

$$
\varphi_{1 \mathrm{D}}^{(\ell)}=\left\{1, \sqrt{3} \xi, \frac{\sqrt{5}}{2}\left(3 \xi^{2}-1\right), \frac{\sqrt{7}}{2}\left(5 \xi^{3}-3 \xi\right), \frac{3}{8}\left(35 \xi^{4}-30 \xi^{2}+3\right)\right\} .
$$

A simple, high-order accurate, and unconditionally stable algorithm to update this solution can developed based on the following two steps:

1. Exactly advect the initial condition over a time step $\Delta t$ :

$$
f(t+\Delta t, x)=f(t, x-v \Delta t)
$$

2. Project this solution back onto the mesh $\mathcal{T}_{i}$.

This process is illustrated in Figure1. These two steps can be compactly written for any starting time $t^{n}$ and final time $t^{n+1}=t^{n}+\Delta t$ as follows:

$$
\begin{aligned}
F_{i}^{(\ell)}\left(t^{n+1}\right) & =\frac{1}{2} \sum_{k=1}^{M} F_{i-1-j}^{(k)}\left(t^{n}\right) \int_{-1}^{-1+2 \nu} \varphi_{1 \mathrm{D}}^{(k)}(\xi+2-2 \nu) \varphi_{1 \mathrm{D}}^{(\ell)}(\xi) d \xi \\
& +\frac{1}{2} \sum_{k=1}^{M} F_{i-j}^{(k)}\left(t^{n}\right) \int_{-1+2 \nu}^{1} \varphi_{1 \mathrm{D}}^{(k)}(\xi-2 \nu) \varphi_{1 \mathrm{D}}^{(\ell)}(\xi) d \xi
\end{aligned}
$$

where

$$
j:=\left\lfloor\frac{v \Delta t}{\Delta x}\right\rfloor \quad \text { and } \quad \nu:=\frac{v \Delta t}{\Delta x}-j .
$$


Here $\lfloor\cdot\rfloor$ denotes the floor operation ${ }^{2}$ and $0 \leq \nu \leq 1$. By construction, update (33) is unconditionally stable independent of the polynomial order of the spatial discretization.

The integrals in equation (33) can be evaluated exactly. For example, in the case of piecewise constants and $j=0, \sqrt{33}$ ) is nothing more than the first-order upwind scheme:

$$
F_{i}^{(1), n+1}=F_{i}^{(1), n}-\nu\left(F_{i}^{(1), n}-F_{i-1}^{(1), n}\right) .
$$

In the case of piecewise linear polynomials and $j=0$, the scheme can be written as follows:

$$
\begin{aligned}
F_{i}^{(1), n+1}=F_{i}^{(1), n}-\nu\left(\left[F_{i}^{(1), n}+\sqrt{3} F_{i}^{(2), n}\right]-\left[F_{i-1}^{(1), n}+\sqrt{3} F_{i-1}^{(2), n}\right]\right) \\
+\sqrt{3} \nu^{2}\left(F_{i}^{(2), n}-F_{i-1}^{(2), n}\right), \\
F_{i}^{(2), n+1}=F_{i}^{(2), n}+\sqrt{3} \nu\left(\left[F_{i}^{(1), n}-\sqrt{3} F_{i}^{(2), n}\right]-\left[F_{i-1}^{(1), n}+\sqrt{3} F_{i-1}^{(2), n}\right]\right) \\
-\sqrt{3} \nu^{2}\left(F_{i}^{(1), n}-F_{i-1}^{(1), n}-2 \sqrt{3} F_{i-1}^{(2), n}\right)+2 \nu^{3}\left(F_{i}^{(2), n}-F_{i-1}^{(2), n}\right) .
\end{aligned}
$$

The above method is a close cousin to the Lax-Wendroff discontinuous Galerkin scheme (LxW-DG) of Qiu, Dumbser, and Shu 35. In particular, in the case of piecewise linear polynomials the LxW-DG for the advection equation can be written as

$$
\begin{aligned}
F_{i}^{(1), \mathrm{n}+1}= & F_{i}^{(1), n}-\nu\left(\left[F_{i}^{(1), n}+\sqrt{3} F_{i}^{(2), n}\right]-\left[F_{i-1}^{(1), n}+\sqrt{3} F_{i-1}^{(2), n}\right]\right) \\
& +\sqrt{3} \nu^{2}\left(F_{i}^{(2), n}-F_{i-1}^{(2), n}\right), \\
F_{i}^{(2), \mathrm{n}+1}= & F_{i}^{(2), n}+\sqrt{3} \nu\left(\left[F_{i}^{(1), n}-\sqrt{3} F_{i}^{(2), n}\right]-\left[F_{i-1}^{(1), n}+\sqrt{3} F_{i-1}^{(2), n}\right]\right) \\
- & 3 \nu^{2}\left(F_{i}^{(2), n}-F_{i-1}^{(2), n}\right) .
\end{aligned}
$$

We note that (38)-(39) and $(36)-(37)$ agree except in the $\nu^{2}$ and $\nu^{3}$ terms in the $F^{(2)}$ update. We argue below that these additional terms in the method given by (36)-(37) are crucial in ensuring stability up to CFL number one, and their absence in the LxW-DG method cause a non-optimal stability result.

Claim. The numerical update given by $\sqrt{36}-\sqrt{37}$ is stable for $0 \leq \nu \leq 1$.

Proof. We apply von Neumann stability analysis to (36)-(37) by assuming the following ansatz:

$$
F_{i}^{(\ell), n}=F^{(\ell), n} e^{I \xi i \Delta x}
$$

\footnotetext{
${ }^{2}$ this function takes a real input and rounds down to the largest integer that is smaller than or equal to the input.
} 
where $I=\sqrt{-1}$. Plugging this into $36-37$ yields

$$
\left[\begin{array}{l}
F^{(1)} \\
F^{(2)}
\end{array}\right]^{n+1}=\left[\begin{array}{cc}
1+\nu(\zeta-1) & \sqrt{3} \nu(1-\nu)(\zeta-1) \\
\sqrt{3} \nu(\nu-1)(\zeta-1) & 1+2 \nu^{3}(1-\zeta)+6 \nu^{2} \zeta-3 \nu(\zeta+1)
\end{array}\right]\left[\begin{array}{l}
F^{(1)} \\
F^{(2)}
\end{array}\right]^{n}
$$

where $\zeta=e^{-I \xi \Delta x}$. With some work, which is omitted here, one can show that the maximum modulus of the eigenvalues of the amplification matrix for $0 \leq \nu \leq 1$ is given by

$$
g(\nu)=\max \left(1,\left|1-6 \nu+6 \nu^{2}\right|\right)
$$

We note that

$$
g(\nu) \equiv 1 \quad \forall \nu \in[0,1]
$$

which concludes the proof.

Claim. The numerical update given by $38-39$ is stable for $0 \leq \nu \leq \frac{1}{3}$.

Proof. Using the same von Neumann ansatz as in the previous claim, this time applied to $38-(39)$, yields

$$
\left[\begin{array}{l}
F^{(1)} \\
F^{(2)}
\end{array}\right]^{n+1}=\left[\begin{array}{cc}
1+\nu(\zeta-1) & \sqrt{3} \nu(1-\nu)(\zeta-1) \\
\sqrt{3} \nu(1-\zeta) & 1-3 \nu(\zeta+1)+3 \nu^{2}(\zeta-1)
\end{array}\right]\left[\begin{array}{l}
F^{(1)} \\
F^{(2)}
\end{array}\right]^{n} .
$$

With some work, which is omitted here, one can show that the maximum modulus of the eigenvalues of the amplification matrix for $0 \leq \nu \leq 1$ is given by

$$
g_{\mathrm{LxW}-\mathrm{DG}}(\nu)=\max (1,|1-6 \nu|)
$$

We note that

$$
g_{\mathrm{LxW}-\mathrm{DG}}(\nu) \equiv 1 \quad \forall \nu \in\left[0, \frac{1}{3}\right], \quad \text { but } \quad g_{\mathrm{LxW}-\mathrm{DG}}(\nu)>1 \quad \forall \nu \in\left(\frac{1}{3}, 1\right]
$$

which concludes the proof. Also note that $g_{\mathrm{LxW}-\mathrm{DG}}(\nu)$ and $g(\nu)$ are the same except for the additional term $6 \nu^{2}$ in $g(\nu)$. Therefore, we conclude that this additional term is crucial in ensuring stability up to CFL number one for the method given by $36-(37)$.

In the above discussion we focused on the piecewise linear DG method. However, more generally, the LxW-DG method behaves similarly to the standard Runge-Kutta DG methods (RK-DG) [16] in that the maximum allowable CFL number is inversely proportional to the polynomial order of the spatial discretization. The modified LxW-DG scheme represented by (36)-(37), and more generally by (33), always has some modified and some additional terms in the update (e.g., the $\nu^{2}$ and $\nu^{3}$ terms in $(37)$ ) that produce a scheme with optimal stability. 
(a)

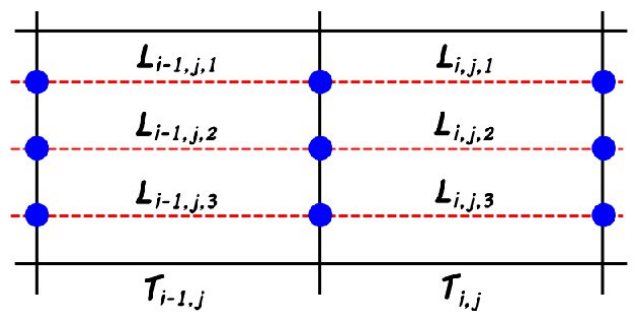

(b)

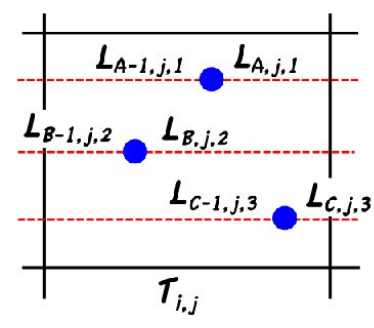

Figure 2: Illustration of the advection algorithm used in the proposed semi-Lagrangian method. Panel (a) illustrates a method with 3 Gaussian quadrature lines in the $x$-direction. The solution along each line segment, e.g., $L_{i, j, 1}$, in each element, e.g., $\mathcal{T}_{i j}$, is a $1 \mathrm{D}$ polynomial. Each Gaussian quadrature line has a different velocity, and as such, each line will get shifted by a different amount; this is shown in Panel (b). The subscript labels $A, B$, and $C$ in Panel (b) highlight the fact that after advection in the $x$-direction, each Gaussian quadrature line might come from a different elements.

\subsection{A semi-Lagrangian DG method for Vlasov-Poisson}

In this section, we describe in detail a semi-Lagrangian discontinuous Galerkin scheme for solving a quasi-1D problem of the form:

$$
f_{, t}+a(v) f_{, x}=0, \quad(t, x, v) \in \mathbb{R}^{+} \times \mathbb{R} \times \mathbb{R} .
$$

This resulting scheme can then be inserted into Steps 1, 3, and 4 of Algorithm 1 for solving the $1+1$ dimensional Vlasov-Poisson system given by (15) and (16). We note that in Steps 1 and $4, a(v)=v$; while in Step 3 the roles of $x$ and $v$ are reversed and $a(x)=E^{n+\frac{1}{2}}(x)$.

We construct a Cartesian grid over $\Omega=[-L, L] \times\left[-V_{\max }, V_{\max }\right]$, with uniform grid spacing $\Delta x$ and $\Delta v$ in each coordinate direction. The mesh element $\mathcal{T}_{i j}$ is centered at the coordinates

$$
x_{i}=-L+\left(i-\frac{1}{2}\right) \Delta x \quad \text { and } \quad v_{j}=-V_{\max }+\left(j-\frac{1}{2}\right) \Delta v .
$$

each element can be mapped to the canonical element via the simple linear transformation:

$$
x=x_{i}+\xi \frac{\Delta x}{2}, \quad v=v_{j}+\eta \frac{\Delta v}{2} .
$$

Next, we further subdivide each element by introducing for each horizontal row of elements, $j$, a set of $M$ horizontal lines located at

$$
v_{j k}:=v_{j}+\eta_{k} \frac{\Delta v}{2}, \quad \text { for } \quad k=1, \ldots, M,
$$

where $\eta_{k}$ are the roots of the $M^{\text {th }}$ degree Legendre polynomial. Along each of these lines, we pretend that we are solving a 1D constant coefficient advection of the form:

$$
f_{, t}+a\left(v_{j k}\right) f_{, x}=0
$$




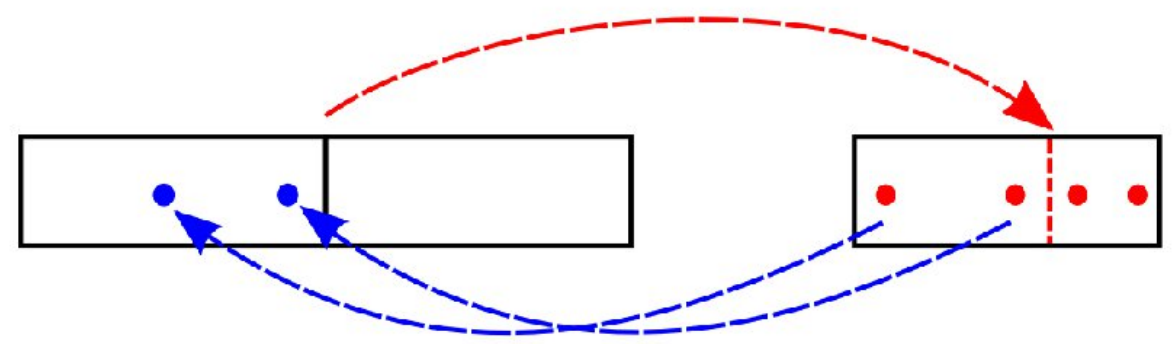

2. Backwards to get solution at quadrature points

Figure 3: Illustration of the forward and backward nature of the proposed semi-Lagrangian scheme. First, the cell edges are propagated forward from their initial time to their final time. Once these locations are known, Gauss-Legendre quadrature points are placed between the old cell edges and the new cell edges. In order to find solution values at these Gauss-Legendre points, we trace backwards along the characteristics to the initial time.

This equation is then essentially solved by the 1D method described in the previous section: $\$ 5.1$. This is depicted in Figure 2, where we have chosen $M=3$ for illustration purposes 3 . Finally, a fully 2D solution is reconstructed in each element, $\mathcal{T}_{i j}$, by summing the $M$ 1D solutions for $k=1, \ldots, M$ with the appropriate Gaussian quadrature weights. This algorithm is summarized in Algorithm 2.

In the semi-Lagrangian way of thinking there are generally two philosophies:

1. Forward evolution: Each "particle" ${ }^{4}$ is advected forward in time and then a solution at the the future time is reconstructed (e.g., Particle-in-cell [27, 7] and Crouseilles et al. [21]).

2. Backward evolution: The solution at a specific location in the future is determined by tracing backwards along characteristics (e.g., Restelli et al. [36]).

We interpret the semi-Lagrangian DG method described in Algorithm 2 as a sort of mixed forward and backward method:

1. Forward evolution phase: The cell edges are propagated forward from their initial time to their final time. This process determines the set of quadrature points necessary for the projection step. This forward evolution step is needed in order to attain mass conservation.

\footnotetext{
${ }^{3}$ The parameter $M$ is chosen to coincide with the spatial order of accuracy of the scheme. In the numerical examples section we always take $M=5$ to coincide with a fifth-order accurate discretization in $x$ and $v$.

${ }^{4}$ A "particle" can be a quadrature point, element interface, etc... depending on the details of the particular method.
} 
$\overline{\text { Algorithm } 2 \text { The proposed semi-Lagrangian algorithm for solving quasi-1D }}$ advection equations of the form $f_{, t}+a(v) f_{, x}=0$.

0 . Start with the current solution:

$$
\left.f^{h}\left(t^{n}, x, v\right)\right|_{\mathcal{T}_{i j}}:=\sum_{\ell=1}^{M(M+1) / 2} F_{i j}^{(\ell)} \varphi^{(\ell)}(\xi, \eta),
$$

where $M$ is the desired order of accuracy in $x$ and $v$. The canonical variables $(\xi, \eta)$ are linearly related to the variables $(x, v)$ via 41 .

1. In each element, construct $M$ horizontal lines given by (42), where $\eta_{k}$ are the $M$ Gauss-Legendre quadrature points. The solution in element $\mathcal{T}_{i j}$ along each one of these lines is

$$
\sum_{\ell=1}^{M(M+1) / 2} F_{i j}^{(\ell)} \varphi^{(\ell)}\left(\xi, \eta_{k}\right)
$$

2. Advect the cell interfaces forward in time through 1D advection along each $v_{j k}$. After forward advection along $v_{j k}$, the $i^{\text {th }}$ cell will contain the old interface $i-\frac{1}{2}-I_{j k}$ and this interface will be located a distance $\Delta x \nu_{j k}$ from the new interface $i-\frac{1}{2}$, where

$$
I_{j k}:=\left\lfloor\frac{a\left(v_{j k}\right) \Delta t}{\Delta x}\right\rfloor \quad \text { and } \quad \nu_{j k}:=\frac{a\left(v_{j k}\right) \Delta t}{\Delta x}-I_{j k} .
$$

This is the forwards phase illustrated in Figure 3 .

3. Next we trace characteristics backwards in time for each $v_{j k}$. The resulting process yields:

$$
\begin{aligned}
S_{i j k}^{(\ell)} & :=\frac{1}{2} \sum_{m=1}^{M(M+1) / 2} F_{i-1-I_{j k} j}^{(m)} \int_{-1}^{-1+2 \nu_{j k}} \varphi^{(m)}\left(\xi+2-2 \nu_{j k}, \eta_{k}\right) \varphi^{(\ell)}\left(\xi, \eta_{k}\right) d \xi \\
& +\frac{1}{2} \sum_{m=1}^{M(M+1) / 2} F_{i-I_{j k} j}^{(m)} \int_{-1+2 \nu_{j k}}^{1} \varphi^{(m)}\left(\xi-2 \nu_{j k}, \eta_{k}\right) \varphi^{(\ell)}\left(\xi, \eta_{k}\right) d \xi
\end{aligned}
$$

where each of the above integrals are evaluated using 1D Gauss-Legendre quadrature rules with $M$ points. This step in the algorithm is the backwards phase illustrated in Figure 3 .

4. Finally, we update the solution by integrating in the vertical direction:

$$
F_{i j}^{(\ell), \text { new }}=\sum_{k=1}^{M} \omega_{k} S_{i j k}^{(\ell)}
$$

where $\omega_{k}$ are the usual Gauss-Legendre quadrature weights for the $M$ GaussLegendre quadrature points $\eta_{k}$. 
2. Backward evolution phase: Once the old cell edge locations are known at the new time, Gauss-Legendre quadrature points are placed between the old cell edges and the new cell edges. In order to find solution values at these Gauss-Legendre points, we trace backwards along the characteristics to the initial time.

This process is illustrated in Figure 3 .

\subsection{Poisson solver}

We describe in this section how to efficiently solve the Poisson equation in Step 2 of the operator splitting approach shown in Algorithm 1. Consider first the 1D Poisson equation on $x \in[a, b]$ with mixed boundary conditions:

$$
\phi_{, x x}=\rho(x)-\rho_{0}, \quad \phi_{, x}(a)=\gamma, \quad \phi(b)=\beta .
$$

We apply to the Poisson equation the so-called local discontinuous Galerkin method (LDG) (see [1, 26] for two reviews of various approaches for solving Poisson equations via the DG method), and rewrite it as a system of two equations:

$$
\begin{aligned}
E_{, x} & =\rho(x)-\rho_{0}, \\
\phi_{, x} & =E(x) .
\end{aligned}
$$

We expand $\phi(x), E(x)$, and $\rho(x)$ on each element as follows:

$$
\left.\left\{\phi^{h}(x), E^{h}(x), \rho^{h}(x)-\rho_{0}\right\}\right|_{\mathcal{T}_{i}}=\sum_{k=1}^{M}\left\{\Phi_{i}^{(k)}, \mathbb{E}_{i}^{(k)}, \mathbb{P}_{i}^{(k)}\right\} \varphi_{1 \mathrm{D}}^{(k)}(\xi)
$$

where $M$ is the desired order of accuracy. $\xi=1$ :

We multiply 50 and 51 each by $\varphi_{1 \mathrm{D}}^{(\ell)}(\xi)$ and integrate from $\xi=-1$ to

$$
\begin{gathered}
\frac{1}{\Delta x}\left[\varphi_{1 \mathrm{D}}^{(\ell)} E^{h}\right]_{-1}^{1}-\frac{1}{\Delta x} \int_{-1}^{1} \varphi_{1 \mathrm{D}, \xi}^{(\ell)} E^{h} d \xi=\mathbb{P}_{i}^{(\ell)} \\
\frac{1}{\Delta x}\left[\varphi_{1 \mathrm{D}}^{(\ell)} \phi^{h}\right]_{-1}^{1}-\frac{1}{\Delta x} \int_{-1}^{1} \varphi_{1 \mathrm{D}, \xi}^{(\ell)} \phi^{h} d \xi=\mathbb{E}_{i}^{(\ell)}
\end{gathered}
$$

Next, we apply the following one-sided rules in order to evaluate $\phi^{h}$ and $E^{h}$ at 
the grid interfaces:

$$
\begin{aligned}
\phi^{h}(-1) & :=\sum_{k=1}^{M} \varphi_{1 \mathrm{D}}^{(k)}(-1) \Phi_{i}^{(k)}=\sum_{k=1}^{M}(-1)^{k+1} \sqrt{2 k-1} \Phi_{i}^{(k)} \\
\phi^{h}(1) & :=\sum_{k=1}^{M} \varphi_{1 \mathrm{D}}^{(k)}(-1) \Phi_{i+1}^{(k)}=\sum_{k=1}^{M}(-1)^{k+1} \sqrt{2 k-1} \Phi_{i+1}^{(k)} \\
E^{h}(-1) & :=\sum_{k=1}^{M} \varphi_{1 \mathrm{D}}^{(k)}(1) \mathbb{E}_{i-1}^{(k)}=\sum_{k=1}^{M} \sqrt{2 k-1} \mathbb{E}_{i-1}^{(k)} \\
E^{h}(1) & :=\sum_{k=1}^{M} \varphi_{1 \mathrm{D}}^{(k)}(1) \mathbb{E}_{i}^{(k)}=\sum_{k=1}^{M} \sqrt{2 k-1} \mathbb{E}_{i}^{(k)}
\end{aligned}
$$

Using these definitions, 53 and (54) can be rewritten as follows:

$$
\begin{array}{r}
\sum_{k=1}^{M} \sqrt{2 k-1} \sqrt{2 \ell-1}\left(\mathbb{E}_{i}^{(k)}+(-1)^{\ell} \mathbb{E}_{i-1}^{(k)}\right)-S_{\ell k} \mathbb{E}_{i}^{(k)}=\Delta x \mathbb{P}_{i}^{(\ell)}, \\
\sum_{k=1}^{M}(-1)^{k+1} \sqrt{2 k-1} \sqrt{2 \ell-1}\left(\Phi_{i+1}^{(k)}+(-1)^{\ell} \Phi_{i}^{(k)}\right)-S_{\ell k} \Phi_{i}^{(k)}=\Delta x \mathbb{E}_{i}^{(\ell)},
\end{array}
$$

where $S$ is an $M \times M$ matrix with entries given by

$$
S_{\ell k}=\int_{-1}^{1} \varphi_{1 \mathrm{D}, \xi}^{(\ell)} \varphi_{1 \mathrm{D}}^{(k)} d \xi
$$

Note that the boundary conditions in 49 imply that

$$
\begin{gathered}
\mathbb{E}^{h}(a)=\gamma \quad \Longrightarrow \quad \sum_{k=1}^{M} \sqrt{2 k-1} \mathbb{E}_{0}^{(k)}=\gamma, \\
\phi^{h}(b)=\beta \quad \Longrightarrow \quad \sum_{k=1}^{M}(-1)^{k+1} \sqrt{2 k-1} \Phi_{m_{x}+1}^{(k)}=\beta,
\end{gathered}
$$

where $m_{x}$ is the number of grid elements. 
Putting everything together, 59 and $(60)$ can be written in matrix form:

$$
\begin{aligned}
& \frac{1}{\Delta x}\left[\begin{array}{ccccc}
A & & & & \\
B & A & & & \\
& B & A & & \\
& & \ddots & \ddots & \\
& & & B & A
\end{array}\right]\left[\begin{array}{c}
\overrightarrow{\mathbb{E}}_{1} \\
\overrightarrow{\mathbb{E}}_{2} \\
\overrightarrow{\mathbb{E}}_{3} \\
\vdots \\
\overrightarrow{\mathbb{E}}_{m_{x}}
\end{array}\right]=\left[\begin{array}{c}
\overrightarrow{\mathbb{P}}_{1}-(-1)^{\ell} \sqrt{2 \ell-1} \gamma(\Delta x)^{-1} \\
\overrightarrow{\mathbb{P}}_{2} \\
\overrightarrow{\mathbb{P}}_{3} \\
\vdots \\
\overrightarrow{\mathbb{P}}_{m_{x}}
\end{array}\right], \\
& \frac{1}{\Delta x}\left[\begin{array}{ccccc}
C & D & & & \\
& C & D & & \\
& & C & \ddots & \\
& & & \ddots & D \\
& & & & C
\end{array}\right]\left[\begin{array}{c}
\vec{\Phi}_{1} \\
\vec{\Phi}_{2} \\
\vec{\Phi}_{3} \\
\vdots \\
\vec{\Phi}_{m_{x}}
\end{array}\right]=\left[\begin{array}{c}
\overrightarrow{\mathbb{E}}_{1} \\
\overrightarrow{\mathbb{E}}_{2} \\
\overrightarrow{\mathbb{E}}_{3} \\
\vdots \\
\overrightarrow{\mathbb{E}}_{m_{x}}-\sqrt{2 \ell-1} \beta(\Delta x)^{-1}
\end{array}\right],
\end{aligned}
$$

where, for example,

$$
\overrightarrow{\mathbb{E}}_{i}=\left(\mathbb{E}_{i}^{(1)}, \ldots, \mathbb{E}_{i}^{(M)}\right)^{T},
$$

and $A, B, C$, and $D$ are $M \times M$ matrices with entries given by:

$$
\begin{aligned}
& A_{\ell k}=\sqrt{2 k-1} \sqrt{2 \ell-1}-S_{\ell k}, \\
& B_{\ell k}=(-1)^{\ell} \sqrt{2 k-1} \sqrt{2 \ell-1}, \\
& C_{\ell k}=(-1)^{k+\ell+1} \sqrt{2 k-1} \sqrt{2 \ell-1}-S_{\ell k}, \\
& D_{\ell k}=(-1)^{k+1} \sqrt{2 k-1} \sqrt{2 \ell-1} .
\end{aligned}
$$

The advantage of this formulation is that equations (64) and 65 are already in lower and upper triangular forms, respectively, and therefore can be easily be solved. The matrices $A$ and $C$ can be easily inverted once at the beginning of the calculation.

\subsubsection{Dirichlet boundary conditions}

The above method can easily be adapted to handle Dirichlet boundary conditions:

$$
\phi(a)=\alpha, \quad \phi(b)=\beta,
$$

by noting that these boundary conditions are equivalent to the mixed BCs in (49) if we carefully choose the parameter $\gamma$ in 49. It can be shown that the correct choice for $\gamma$ is given by

$$
\begin{aligned}
\gamma & =\frac{\beta-\alpha}{b-a}+\frac{1}{b-a} \int_{a}^{b}(s-b)\left(\rho(s)-\rho_{0}\right) d s \\
& =\frac{\beta-\alpha}{b-a}+\frac{\Delta x}{b-a} \sum_{i=1}^{m_{x}}\left(x_{i}-b\right) \mathbb{P}_{i}^{(1)}+\frac{\Delta x^{2}}{2 \sqrt{3}(b-a)} \sum_{i=1}^{m_{x}} \mathbb{P}_{i}^{(2)},
\end{aligned}
$$

where $m_{x}$ is the number of grid elements. 


\subsubsection{Periodic boundary conditions}

Periodic boundary conditions can also be readily handled:

$$
\phi(a)=\phi(b), \quad E(a)=E(b),
$$

by again noting that we need to carefully choose $\beta$ and $\gamma$ in 449 . It can be shown that the correct choice for $\gamma$ is given by

$$
\gamma=\frac{1}{b-a} \int_{a}^{b} s\left(\rho(s)-\rho_{0}\right) d s=\frac{\Delta x}{b-a} \sum_{i=1}^{m_{x}} x_{i} \mathbb{P}_{i}^{(1)}+\frac{\Delta x^{2}}{2 \sqrt{3}(b-a)} \sum_{i=1}^{m_{x}} \mathbb{P}_{i}^{(2)},
$$

and $\beta$ is arbitrary. Without loss of generality we simply take $\beta=0$. By solving (49) with $\gamma$ given by $(74)$ and with $\beta=0$, we obtain a solution $\phi(x)$ with the property that

$$
\phi(a)=\phi(b)=0 .
$$

\subsection{High-order split semi-Lagrangian method}

Now that the basic pieces are in place (i.e., a semi-Lagrangian solver for each split-piece $\$ 5.2$ and the Poisson-solver $\$ 5.3$, we are ready to introduce a fully fourth-order accurate method for the Vlasov-Poisson system. We describe in this section some important implementation details needed to achieve this. The resulting method is summarized in Algorithm 3

\subsubsection{Fourth-order operator splitting}

Consider a time-dependent problem where the right-hand side is written as the sum of two differential operators $\mathcal{A}$ and $\mathcal{B}$ :

$$
q_{, t}=\mathcal{A}(q)+\mathcal{B}(q) .
$$

A fourth-order accurate operator splitting technique for such systems was developed by Forest and Ruth [23] and by Yoshida [41, 42]. If we define the following two constants:

$$
\begin{aligned}
& \gamma_{1}=\frac{1}{2-2^{1 / 3}} \approx 1.351207191959658, \\
& \gamma_{2}=-\frac{2^{1 / 3}}{2-2^{1 / 3}} \approx-1.702414383919315,
\end{aligned}
$$


then the fourth-order splitting approach of [23, 41, 42, can be written as a composition of the following seven stages:

$$
\begin{array}{ll}
\text { Stage 1: } & \frac{\gamma_{1} \Delta t}{2} \approx 0.6756 \Delta t \quad \text { step on } \quad q_{, t}=\mathcal{A}(q), \\
\text { Stage 2: } & \gamma_{1} \Delta t \approx 1.3512 \Delta t \quad \text { step on } \quad q_{, t}=\mathcal{B}(q), \\
\text { Stage 3: } & \frac{\left(\gamma_{1}+\gamma_{2}\right) \Delta t}{2} \approx-0.1756 \Delta t \quad \text { step on } \quad q_{, t}=\mathcal{A}(q), \\
\text { Stage 4: } & \gamma_{2} \Delta t \approx-1.7024 \Delta t \quad \text { step on } \quad q_{, t}=\mathcal{B}(q), \\
\text { Stage 5: } & \frac{\left(\gamma_{1}+\gamma_{2}\right) \Delta t}{2} \approx-0.1756 \Delta t \quad \text { step on } \quad q_{, t}=\mathcal{A}(q), \\
\text { Stage 6: } & \gamma_{1} \Delta t \approx 1.3512 \Delta t \quad \text { step on } \quad q_{, t}=\mathcal{B}(q), \\
\text { Stage 7: } & \frac{\gamma_{1} \Delta t}{2} \approx 0.6756 \Delta t \text { step on } \quad q_{, t}=\mathcal{A}(q) .
\end{array}
$$

We note that this splitting approach requires some steps larger than $\Delta t$ : Step 2 and Step 6; as well as backward steps: Step 3, Step 4, and Step 5.

\subsubsection{Application to Vlasov-Poisson}

One difficulty with raising the temporal order of accuracy from two to four is the time-dependence of the electric field. In other words, the Cheng and Knorr 9 method does not completely reduce the Vlasov-Poisson to two constant coefficient problems, since the electric field remains time-dependent. In the case of Strang splitting, it turned out that one could easily generate a second order accurate representation of the electric field at the half time step, $t^{n}+\frac{1}{2} \Delta t$, as required in Step 3 of Algorithm 1, simply by carrying out Steps 1 and 2 of Algorithm 1. Additional attention must be paid in order to obtain temporally fourth-order accurate representations of the electric field.

In order to avoid having to use the electric field at different points in time (i.e., a multi-step method), we construct the fourth-order Taylor polynomial centered at $t=t^{n}$ :

$$
\overline{\mathbf{E}}(t, \mathbf{x}):=\mathbf{E}^{n}+\left(t-t^{n}\right) \mathbf{E}_{, t}^{n}+\frac{1}{2}\left(t-t^{n}\right)^{2} \mathbf{E}_{, t t}^{n}+\frac{1}{6}\left(t-t^{n}\right)^{3} \mathbf{E}_{, t t t}^{n} .
$$

The electric field value $\mathbf{E}^{n}$ is computed from the Poisson equation:

$$
\nabla \cdot \mathbf{E}^{n}=\nabla^{2} \phi^{n}=\rho^{n}-\rho_{0} .
$$

The first time derivative of the electric field is proportional to the momentum:

$$
\nabla \cdot \mathbf{E}_{, t}=\rho_{, t}=-\nabla \cdot(\rho \mathbf{u}) \quad \Longrightarrow \quad \mathbf{E}_{, t}^{n}=-(\rho \mathbf{u})^{n},
$$

and is thus readily computable. In order to compute the remaining time derivatives, we write down the evolution equations for the first few moments of the Vlasov-Poisson equation:

$$
\begin{aligned}
\rho_{, t}+\nabla \cdot(\rho \mathbf{u}) & =0, \\
(\rho \mathbf{u})_{, t}+\nabla \cdot \mathbb{E} & =\rho \mathbf{E}, \\
\mathbb{E}_{, t}+\nabla \cdot \mathbb{F} & =\rho(\mathbf{u} \mathbf{E}+\mathbf{E u}),
\end{aligned}
$$


where

$$
\rho:=\int_{\mathbf{v}} f d \mathbf{v}, \quad \rho \mathbf{u}:=\int_{\mathbf{v}} \mathbf{v} f d \mathbf{v}, \quad \mathbb{E}:=\int_{\mathbf{v}} \mathbf{v} \mathbf{v} f d \mathbf{v}, \quad \text { and } \quad \mathbb{F}:=\int_{\mathbf{v}} \mathbf{v} \mathbf{v} \mathbf{v} f d \mathbf{v} .
$$

Using equation 80 and the above moment evolution equations, we can compute the second and third time derivatives of the electric field entirely in terms of spatial derivatives:

$$
\begin{aligned}
\mathbf{E}_{, t t} & =-(\rho \mathbf{u})_{, t}=\nabla \cdot \mathbb{E}-\rho \mathbf{E}, \\
\mathbf{E}_{, t t t} & =\nabla \cdot \mathbb{E}_{, t}-\rho_{, t} \mathbf{E}-\rho \mathbf{E}_{, t} \\
& =\nabla \cdot(\rho \mathbf{u} \mathbf{E}+\rho \mathbf{E u})-\nabla \cdot \nabla \cdot \mathbb{F}+\mathbf{E} \nabla \cdot(\rho \mathbf{u})+\rho^{2} \mathbf{u} .
\end{aligned}
$$

It is clear from these expressions that in order to compute $\mathbf{E}_{, t t}$ and $\mathbf{E}_{, t t t}$, we need to be able to compute first and second derivatives in space. One approach for doing this in the discontinuous Galerkin framework is to multiply by a test function and then integrate-by-parts. However, this approach will in general lead to a loss of accuracy. Instead, the approach taken in this work is to apply central finite differences that work directly on the Legendre coefficients of the function that needs to be differentiated.

Consider the $L_{2}$-projection of the function $f(x)$, where $f: \mathbb{R} \rightarrow \mathbb{R}$, onto the space of piecewise polynomials of degree four on a uniform mesh of elements, $\mathcal{T}_{i}$, that each have width $\Delta x$ :

$$
\left.f^{h}\right|_{\mathcal{T}_{i}}=\sum_{\ell=1}^{5} F_{i}^{(\ell)} \varphi_{1 \mathrm{D}}^{(\ell)}(\xi)
$$

Therefore, $f^{h}$ represents the finite dimensional approximation of $f(x)$. We can approximate the first and second derivatives of $f(x)$ to $\mathcal{O}\left(\Delta x^{5}\right)$ accuracy by computing appropriate central finite differences of the Legendre cofficients $F^{(\ell)}$. If we let $D_{x} f^{h}$ and $D_{x x} f^{h}$ represent the finite dimensional approximations of $f^{\prime}(x)$ and $f^{\prime \prime}(x)$, respectively, then the central finite difference formulas on the Legendre coefficients are

$$
\begin{gathered}
{\left[\begin{array}{c}
D_{x} F_{i}^{(1)} \\
D_{x} F_{i}^{(2)} \\
D_{x} F_{i}^{(3)} \\
D_{x} F_{i}^{(4)} \\
D_{x} F_{i}^{(5)}
\end{array}\right]=\frac{1}{2 \Delta x}\left[\begin{array}{c}
\Delta_{1} F_{i}^{(1)}-2 \sqrt{5} \Delta_{1} F_{i}^{(3)}+78 \Delta_{1} F_{i}^{(5)} \\
\Delta_{1} F_{i}^{(2)}-\frac{10}{3} \sqrt{3} \sqrt{7} \Delta_{1} F_{i}^{(4)} \\
\Delta_{1} F_{i}^{(3)}-14 \sqrt{5} \Delta_{1} F_{i}^{(5)} \\
\Delta_{1} F_{i}^{(4)} \\
\Delta_{1} F_{i}^{(5)}
\end{array}\right],} \\
{\left[\begin{array}{c}
D_{x x} F_{i}^{(1)} \\
D_{x x} F_{i}^{(2)} \\
D_{x x} F_{i}^{(3)} \\
D_{x x} F_{i}^{(4)} \\
D_{x x} F_{i}^{(5)}
\end{array}\right]=\frac{1}{\Delta x^{2}}\left[\begin{array}{c}
\Delta_{2} F_{i}^{(1)}-\sqrt{5} \Delta_{2} F_{i}^{(3)}+11 \Delta_{2} F_{i}^{(5)} \\
\Delta_{2} F_{i}^{(2)}-\frac{5}{3} \sqrt{3} \sqrt{7} \Delta_{2} F_{i}^{(4)} \\
\Delta_{2} F_{i}^{(3)}-7 \sqrt{5} \Delta_{2} F_{i}^{(5)} \\
\Delta_{2} F_{i}^{(4)} \\
\Delta_{2} F_{i}^{(5)}
\end{array}\right],}
\end{gathered}
$$




\begin{tabular}{|c||c|c||c|c|}
\hline Mesh & $f^{\prime}(x)$ error & $\log _{2}$ (Ratio) & $f^{\prime \prime}(x)$ error & $\log _{2}$ (Ratio) \\
\hline \hline 25 & $1.747 \times 10^{-4}$ & - & $8.292 \times 10^{-5}$ & - \\
\hline 50 & $5.543 \times 10^{-6}$ & 4.98 & $2.672 \times 10^{-6}$ & 4.96 \\
\hline 100 & $1.738 \times 10^{-7}$ & 5.00 & $8.413 \times 10^{-8}$ & 4.99 \\
\hline 200 & $5.437 \times 10^{-9}$ & 5.00 & $2.634 \times 10^{-9}$ & 5.00 \\
\hline 400 & $1.699 \times 10^{-10}$ & 5.00 & $8.364 \times 10^{-11}$ & 4.98 \\
\hline
\end{tabular}

Table 1: Relative $L_{2}$-norm errors for computing $f^{\prime}(x)$ and $f^{\prime \prime}(x)$ using the Legendre coefficient finite difference formulas 87 and 88, respectively. The example shown here is for $f(x)=$ $e^{\sin (2 \pi x)}$ on a uniform mesh on $0 \leq x \leq 1$. Periodic boundary conditions are imposed to compute the derivative in the first and last elements: $F_{0}^{(k)}=F_{M}^{(k)}$ and $F_{M+1}^{(k)}=F_{1}^{(k)}$ for each $k=1,2,3,4,5$.

where

$$
\begin{aligned}
& \Delta_{1} F_{i}^{(k)}:=F_{i+1}^{(k)}-F_{i-1}^{(k)}, \\
& \Delta_{2} F_{i}^{(k)}:=F_{i+1}^{(k)}-2 F_{i}^{(k)}+F_{i-1}^{(k)} .
\end{aligned}
$$

To the best of our knowledge, this is the first time such formulas have been written down in the context of discontinuous Galerkin methods. In Table 1 we verify the order of accuracy by computing the first and second derivatives of $f(x)=e^{\sin (2 \pi x)}$. The errors in this table are computed using the relative $L_{2}$ errors defined by equation (B.4) with $M=5$ and varying $\Delta x$. See Appendix B for more details.

Once we have constructed an approximation to the time-dependent electric field, we are faced with an advection equation with time-dependent coefficients:

$$
f_{, t}+\overline{\mathbf{E}}(t, \mathbf{x}) \cdot f_{, \mathbf{v}}=0 .
$$

This equation can be readily solved to high-order via the method of characteristics. The key step in this approach is the evolution of the coordinates $\mathbf{v}$ as function of time:

$$
\begin{aligned}
& \frac{d \mathbf{v}}{d t}=\overline{\mathbf{E}}(t, \mathbf{x}) \Longrightarrow \mathbf{v}\left(t^{n}+\Delta t\right)=\mathbf{v}\left(t^{n}\right)+\int_{t^{n}}^{t^{n}+\Delta t} \overline{\mathbf{E}}(t, \mathbf{x}) d t \\
& \mathbf{v}\left(t^{n}+\Delta t\right)=\mathbf{v}\left(t^{n}\right)+\Delta t \mathbf{E}^{n}+\frac{\Delta t^{2}}{2} \mathbf{E}_{, t}^{n}+\frac{\Delta t^{3}}{6} \mathbf{E}_{, t t}^{n}+\frac{\Delta t^{4}}{24} \mathbf{E}_{, t t t}^{n} .
\end{aligned}
$$

In other words, the semi-Lagrangian DG method as outlined in 5.2 remains largely unaltered by the fact that the electric is time dependent. The only difference is that interfaces and quadrature points are transported by equation (93) instead of the simpler version of this equation when $\overline{\mathbf{E}}$ is constant in time.

We note than an important advantage of this approach is that, just as with Strang splitting, it requires only a single Poisson solver per time step. Finally, we 
summarize the complete fourth-order splitting method for the Vlasov-Poisson system in Algorithm 3 .

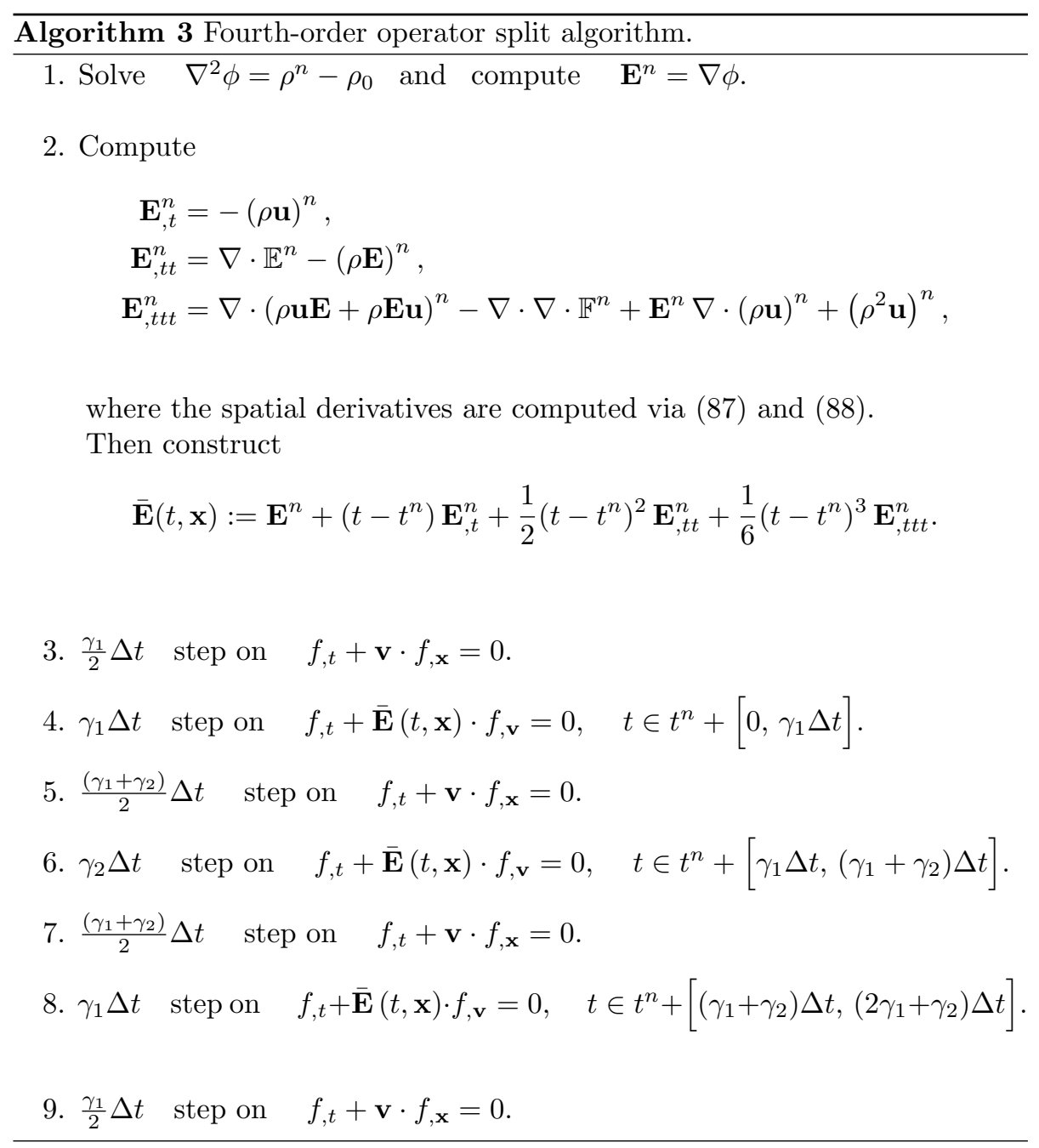

\subsection{Mass conservation and positivity in the mean}

The description of the method so far has yielded a fourth-order accurate in time and fifth-order in space semi-Lagrangian method for the Vlasov-Poisson equations. It still remains to show that the method is mass conservative and that it is positivity-preserving. We prove mass conservation in the first theorem below. This is followed by a proof that each step in the operator split semi- 
Lagrangian produces a solution that it is positive in the mean $n^{5}$

Theorem 1 (Conservation). Each step of the semi-Lagrangian method described above is mass conservative.

Proof. It suffices to show that the semi-Lagrangian scheme is conservative on the quasi-1D problem given by 40 with periodic boundary conditions in $x$. Using the notation of Algorithm 2 the update for the mean value in cell $\mathcal{T}_{i j}$ can be written in the form:

$$
\begin{aligned}
F_{i j}^{(1), \text { new }} & =\sum_{k=1}^{M} \omega_{k} S_{i j k}^{(1)} \\
& =\frac{1}{2} \sum_{k=1}^{M} \sum_{m=1}^{M(M+1) / 2} \omega_{k} F_{i-1-I_{j k} j}^{(m)} \int_{-1}^{-1+2 \nu_{j k}} \varphi^{(m)}\left(\xi+2-2 \nu_{j k}, \eta_{k}\right) d \xi \\
& +\frac{1}{2} \sum_{k=1}^{M} \sum_{m=1}^{M(M+1) / 2} \omega_{k} F_{i-I_{j k} j}^{(m)} \int_{-1+2 \nu_{j k}}^{1} \varphi^{(m)}\left(\xi-2 \nu_{j k}, \eta_{k}\right) d \xi .
\end{aligned}
$$

The total integral of $f^{h}(t, x, v)$ over the entire computational domain can then be written as

$$
\begin{aligned}
\sum_{i, j} F_{i j}^{(1), \text { new }} & =\frac{1}{2} \sum_{i, j, k, m} \omega_{k} F_{i-1-I_{j k} j}^{(m)} \int_{-1}^{-1+2 \nu_{j k}} \varphi^{(m)}\left(\xi+2-2 \nu_{j k}, \eta_{k}\right) d \xi \\
& +\frac{1}{2} \sum_{i, j, k, m} \omega_{k} F_{i-I_{j k} j}^{(m)} \int_{-1+2 \nu_{j k}}^{1} \varphi^{(m)}\left(\xi-2 \nu_{j k}, \eta_{k}\right) d \xi .
\end{aligned}
$$

We make the following change of variables in the integrals above:

$$
s=\xi+2-2 \nu_{j k} \quad \text { and } \quad s=\xi-2 \nu_{j k},
$$

respectively, which yields:

$$
\begin{aligned}
\sum_{i, j} F_{i j}^{(1), \text { new }} & =\frac{1}{2} \sum_{i, j, k, m} \omega_{k} F_{i-1-I_{j k} j}^{(m)} \int_{1-2 \nu_{j k}}^{1} \varphi^{(m)}\left(s, \eta_{k}\right) d s \\
& +\frac{1}{2} \sum_{i, j, k, m} \omega_{k} F_{i-I_{j k} j}^{(m)} \int_{-1}^{1-2 \nu_{j k}} \varphi^{(m)}\left(s, \eta_{k}\right) d s .
\end{aligned}
$$

Since we are summing over all $i$, we shift the first index of $F$ without changing the total sum; this step allows us to combine the two integrals into one:

$$
\sum_{i, j} F_{i j}^{(1), \text { new }}=\frac{1}{2} \sum_{i, j, k, m} \omega_{k} F_{i j}^{(m)} \int_{-1}^{1} \varphi^{(m)}\left(s, \eta_{k}\right) d s .
$$

\footnotetext{
${ }^{5}$ We show in the next subsection how to turn positivity in the mean into global positivity.
} 
Since $\varphi^{(m)}(s, \eta)$ is polynomial of degree at most $M-1$ in $\eta$, the Gaussian quadrature represented by the sum over $k$ is exact:

$$
\begin{aligned}
\sum_{i, j} F_{i j}^{(1), \text { new }} & =\sum_{i, j} \sum_{m} F_{i j}^{(m)}\left[\frac{1}{4} \int_{-1}^{1} \int_{-1}^{1} \varphi^{(m)}(s, \eta) d s d \eta\right] \\
& =\sum_{i, j} \sum_{m} F_{i j}^{(m)} \delta_{1 m}=\sum_{i, j} F_{i j}^{(1)}
\end{aligned}
$$

where $\delta_{1 m}$ is the Kronecker delta.

Theorem 2 (Positivity in the mean). Let $M$ denote the spatial order of accuracy and let

$$
K:=\left\lceil\frac{M}{2}\right\rceil,
$$

where $\lceil\cdot\rceil$ denotes the ceiling operation ${ }^{6}$. Let $f^{h}\left(t^{n}, x, v\right)$ be a function defined on the broken finite element space (22) with $q=M-1$, and let

$$
\tilde{f}_{i j}^{h}\left(t^{n}, \xi, \eta\right):=\left.f^{h}\left(t^{n}, x, v\right)\right|_{\mathcal{T}_{i j}},
$$

where $(\xi, \eta) \in[-1,1] \times[-1,1]$ are the variables on the canonical element. Assume that $\tilde{f}_{i j}^{h}\left(t^{n}, \xi, \eta\right)$ is non-negative at all of the following $2 M K$ points:

$$
\begin{aligned}
& (\xi, \eta)=\left(\xi_{\ell j k}^{L}, \eta_{k}\right), \quad \text { where } \xi_{\ell j k}^{L}:=\nu_{j k}\left(1-s_{\ell}\right)+s_{\ell}, \\
& (\xi, \eta)=\left(\xi_{\ell j k}^{R}, \eta_{k}\right), \quad \text { where } \xi_{\ell j k}^{R}:=\nu_{j k}\left(1+s_{\ell}\right)-1,
\end{aligned}
$$

$\forall k=1, \ldots, M$ and $\forall \ell=1, \ldots, K$. In the above expression, $\nu_{j k}$ is given by (46), se denotes the $\ell^{\text {th }}$ quadrature point in the standard $1 D$ Gauss-Legendre rule with $K$ points, and $\eta_{k}$ denotes the $k^{\text {th }}$ quadrature point in the standard $1 D$ Gauss-Legendre rule with $M$ points.

If one time-step in one coordinate direction is taken using the semi-Lagrangian scheme as described in Algorithm 2 with $f^{h}\left(t^{n}, x, v\right)$ as the initial condition, then the approximate solution at the end of this time-step will have a non-negative average in every element (independent of the time step $\Delta t$ ):

$$
F_{i j}^{(1), \text { new }} \geq 0, \quad \forall \mathcal{T}_{i j} \in \Omega^{h} .
$$

Proof. Using the notation of Algorithm 2, the update for the mean-value in element $\mathcal{T}_{i j}$ can be written as

$$
\begin{aligned}
F_{i j}^{(1), \text { new }} & =\frac{1}{2} \sum_{k=1}^{M} \omega_{k} \int_{-1}^{-1+2 \nu_{j k}}\left\{\sum_{m=1}^{M(M+1) / 2} F_{i-1-I_{j k} j}^{(m)} \varphi^{(m)}\left(\xi+2-2 \nu_{j k}, \eta_{k}\right)\right\} d \xi \\
& +\frac{1}{2} \sum_{k=1}^{M} \omega_{k} \int_{-1+2 \nu_{j k}}^{1}\left\{\sum_{m=1}^{M(M+1) / 2} F_{i-I_{j k} j}^{(m)} \varphi^{(m)}\left(\xi-2 \nu_{j k}, \eta_{k}\right)\right\} d \xi .
\end{aligned}
$$

\footnotetext{
${ }^{6}$ this function takes a real input and rounds up to the smallest integer that is larger than or equal to the input.
} 
We note that the terms inside the brackets are simply shifted solution values, allowing us to express the above update as follows:

$$
F_{i j}^{(1), \text { new }}=\frac{1}{2} \sum_{k=1}^{M} \omega_{k}\left\{\int_{-1}^{-1+2 \nu_{j k}} P_{i j k}^{R}(\xi) d \xi+\int_{-1+2 \nu_{j k}}^{1} P_{i j k}^{L}(\xi) d \xi\right\},
$$

where

$$
\begin{array}{ll}
P_{i j k}^{R}(\xi):=\tilde{f}_{i-1-I_{j k} j}^{h}\left(t^{n}, \xi+2-2 \nu_{j k}, \eta_{k}\right) & \text { for } \quad \xi \in\left[-1,-1+2 \nu_{j k}\right], \\
P_{i j k}^{L}(\xi):=\tilde{f}_{i-I_{j k} j}^{h}\left(t^{n}, \xi-2 \nu_{j k}, \eta_{k}\right) & \text { for } \quad \xi \in\left[-1+2 \nu_{j k}, 1\right] .
\end{array}
$$

Since $P_{i j k}^{L}(\xi)$ and $P_{i j k}^{R}(\xi)$ are polynomials of degree at most $M-1$, we can exactly evaluate each of the above integrals via Gauss-Legendre quadrature rules using $K$ points (where $K$ is defined in $(94)$ ):

$$
F_{i j}^{(1) \text { new }}=\frac{1}{2} \sum_{k=1}^{M} \omega_{k}\left\{\sum_{\ell=1}^{K} \varpi_{\ell} P_{i j k}^{R}\left(\xi_{\ell j k}^{R}\right)+\sum_{\ell=1}^{K} \varpi_{\ell} P_{i j k}^{L}\left(\xi_{\ell j k}^{L}\right)\right\},
$$

where the $\varpi_{\ell}$ 's are the standard quadrature weights for Gauss-Legendre quadrature with $K$ points.

To conclude our proof, we note that since all of the quadrature weights in the above expression for $F_{i j}^{(1), \text { new }}$ are strictly positive, we obtain positivity in the mean,

$$
F_{i j}^{(1), \text { new }} \geq 0
$$

if $\tilde{f}_{i j}^{h}\left(t^{n}, \xi, \eta\right)$ is non-negative at all of the $2 M K$ points defined in 96-97.

\subsection{Positivity-preserving limiter}

One of the key assumptions in the above proof of positivity in the mean is the fact that solution prior to a time-step must be positive at all of points defined in (96)-(97). We show in this subsection how to the limit the solutions, including the initial condition, so that we achieve positivity at all of these points. The key piece of technology necessary for achieving this positivity is a modification of the limiter of Zhang and Shu [44. This limiter is simple to implement and is completely local to each element.

The solution on some element $\mathcal{T}$ can be written as

$$
f^{h}(\xi, \eta):=\sum_{\ell=1}^{M(M+1) / 2} F^{(\ell)} \varphi^{(\ell)}(\xi, \eta),
$$

where $M$ is the desired order of accuracy in space. We assume that the element average is non-negative: $F^{(1)} \geq 0$. We sample this solution on a set of test points:

$$
\left(\xi_{i}, \eta_{i}\right) \in[-1,1] \times[-1,1] \quad \text { for } \quad i=1,2, \ldots, P,
$$


and define:

$$
m:=\min _{i=1, \ldots, P} f^{h}\left(\xi_{i}, \eta_{i}\right) .
$$

Note that $m \in\left(-\infty, F^{(1)}\right]$.

The limited solution is defined as follows:

$$
\tilde{f}^{h}(\xi, \eta):=F^{(1)}+\theta \cdot\left(f^{h}(\xi, \eta)-F^{(1)}\right),
$$

where

$$
\theta=\min \left\{1, \frac{F^{(1)}}{F^{(1)}-m}\right\} .
$$

Note that $0 \leq \theta \leq 1$ and that

$$
\theta=\left\{\begin{array}{lll}
1 & \text { if } & 0 \leq m \leq F^{(1)} \\
\in[0,1) & \text { if } & m<0 .
\end{array}\right.
$$

This means that if the solution is already non-negative at each of the test points, then this limiter does not alter the solution. On the other hand, if the solution on the element is negative at any of the test points, then the high-order corrections are damped until the solution is again non-negative. We are guaranteed that as $\theta \rightarrow 0$, the solution will eventually become non-negative on the entire element since $F^{(1)} \geq 0$.

In practice we implement the limiting strategy as follows:

1. During each of the stages labelled 3-9 in Algorithm 3, we apply the positivity limiter with the test points given by $(96)-97)$. In this case $P=2 M K$, where $M$ is the desired order of accuracy in space and $K$ is defined by (94). As proved in Theorem 2, this guarantees that in each stage the approximate solution remains positive in the mean.

2. After all of the stages of Algorithm 3 have been carried out, we apply the positivity limiter one more time to the solution, this time with the test points taken as the $P=M^{2}$ Gauss-Legendre quadrature points on $[-1,1] \times[-1,1]$. This final limiting provides some additional positivity enforcement and allows us to compute a variety of integrals of the form (A.1) with function values that are non-negative. This is particularly useful in computing the $L_{1}$-norm A.3, the total energy A.5, and the entropy A.6.

\section{Numerical examples}

In this section we apply the proposed scheme to a variety of numerical test cases. We begin in $\$ 6.1$ by considering a linear advection equation with a velocity field that produces solid body rotation. This example is primarily used to show the benefits of switching from second to fourth-order operator splitting strategies. In $\$ 6.2$ we verify the order of accuracy of the method on a forced Vlasov-Poisson equation where we know the exact solution. In the subsequent 
three subsection we consider three standard problems for the Vlasov-Poisson system: 6.3 the two-stream instability, 6.4 weak Landau damping, and 6.5 strong Landau damping. Unless otherwise stated, all simulations below are done with $5^{\text {th }}$ order in space and with the positivity-preserving limiters as described in $\$ 5.6$ turned on.

\subsection{Linear advection}

We first consider a linear advection under a divergence-free velocity field:

$$
q_{, t}+\mathbf{u} \cdot \nabla q=0 .
$$

We take the computational domain to be $[0,1] \times[0,1]$ and the velocity field to be solid body rotation around $(0.5,0.5)$ :

$$
\mathbf{u}=(u(y), v(x))=(\pi(2 y-1), \pi(1-2 x))^{T} .
$$

The initial condition is taken to be a smooth, compactly supported bump that is centered at $\left(x_{0}, y_{0}\right)=(0.4,0.5)$ :

$$
q(0, x, y)= \begin{cases}\cos ^{6}\left(\frac{5 \pi}{3} r\right) & \text { if } r \leq 0.3 \\ 0 & \text { otherwise }\end{cases}
$$

where

$$
r=\sqrt{\left(x-x_{0}\right)^{2}+\left(y-y_{0}\right)^{2}} .
$$

This problem, just as the Vlasov-Poisson system, is solved via operator splitting on the two operators:

$$
\begin{array}{ll}
\text { Problem } \mathcal{A}: & q_{, t}+u(y) q_{, x}=0, \\
\text { Problem } \mathcal{B}: & q_{, t}+v(x) q_{, y}=0 .
\end{array}
$$

We run the initial condition out to time $t=1$, at which point it should return to its initial state. The errors are computed using the relative $L_{2}$ errors defined by equation (C.4 with $M=5$ and varying $\Delta x=\Delta y$. See Appendix C for

more details. Convergence studies with Strang and the fourth-order operator splitting results are shown in Table 2.

\subsection{A forced problem: verifying order of accuracy}

Next we consider an example of a forced Vlasov-Poisson equation where we have an exact solution. The forced Vlasov-Poisson system is

$$
\begin{aligned}
& f_{, t}+v f_{, x}+E f_{, v}=\psi(t, x, v), \\
& E_{, x}=\int_{-\infty}^{\infty} f(t, x, v) d v-\sqrt{\pi},
\end{aligned}
$$




\begin{tabular}{|c||c|c||c|c|}
\hline Mesh & SL2 Error & $\log _{2}$ (Ratio) & SL4 Error & $\log _{2}$ (Ratio) \\
\hline \hline $10^{2}$ & $3.215 \times 10^{-1}$ & - & $5.679 \times 10^{-1}$ & - \\
\hline $20^{2}$ & $7.185 \times 10^{-2}$ & 2.16 & $3.113 \times 10^{-2}$ & 4.19 \\
\hline $40^{2}$ & $1.578 \times 10^{-2}$ & 2.19 & $1.691 \times 10^{-3}$ & 4.20 \\
\hline $80^{2}$ & $3.923 \times 10^{-3}$ & 2.01 & $1.010 \times 10^{-4}$ & 4.07 \\
\hline $160^{2}$ & $9.890 \times 10^{-4}$ & 1.99 & $6.220 \times 10^{-6}$ & 4.02 \\
\hline $320^{2}$ & $2.454 \times 10^{-4}$ & 2.01 & $3.843 \times 10^{-7}$ & 4.02 \\
\hline $640^{2}$ & $6.136 \times 10^{-5}$ & 2.00 & $2.390 \times 10^{-8}$ & 4.01 \\
\hline
\end{tabular}

Table 2: Convergence study for the linear advection equation. Shown are the relative errors computed via C.4 at time $t=1$. All calculations were done with $5^{\text {th }}$ order accuracy in space using the positivity-preserving limiters and a CFL number of 5.00, where CFL := $\Delta t \max \left\{\max _{y}|u(y)| / \Delta x, \max _{x}|v(x)| / \Delta y\right\}$. SL2 refers to the Strang split semi-Lagrangian scheme and SL4 to the fourth-order split semi-Lagrangian scheme.

on $(t, x, v) \in[0, \infty) \times[-\pi, \pi] \times(-\infty, \infty)$ with periodic boundary conditions in $x$. We take the following source term:

$$
\begin{aligned}
\psi(t, x, v)=\frac{1}{2} \sin (2 x-2 \pi t) e^{-\frac{1}{4}(4 v-1)^{2}}\{ & (2 \sqrt{\pi}+1)(4 v-2 \sqrt{\pi}) \\
& -\sqrt{\pi}(4 v-1) \cos (2 x-2 \pi t)\} .
\end{aligned}
$$

The exact solution in this case is

$$
\begin{aligned}
f(t, x, v) & =\{2-\cos (2 x-2 \pi t)\} e^{-\frac{1}{4}(4 v-1)^{2}}, \\
E(t, x) & =-\frac{\sqrt{\pi}}{4} \sin (2 x-2 \pi t) .
\end{aligned}
$$

The numerical scheme for this forced problem is the same as the one described in $\$ 5$ with two minor modifications. First, the two operators in the operator split scheme are

$$
\begin{array}{ll}
\text { Problem } \mathcal{A}: & f_{, t}+v f_{, x}=\psi(t, x, v), \\
\text { Problem } \mathcal{B}: & f_{, t}+E(t, x) f_{, v}=0,
\end{array}
$$

which means that Problem $\mathcal{A}$ is slightly modified from the unforced VlasovPoisson system. The modified $\mathcal{A}$ still has the same characteristics as the case with no source term; the only difference is that the solution is no longer constant along the characteristics. In order to advance $f$ forward under the influence of operator $\mathcal{A}$, we use the method of characteristics and obtain the following solution:

$$
f\left(t^{n+1}, x, v\right)=f\left(t^{n}, x-v \Delta t, v\right)+\int_{t^{n}}^{t^{n+1}} \psi\left(s, x+v\left(s-t^{n+1}\right), v\right) d s .
$$


The time integral in the above expression can be easily exactly evaluated; we omit the details here.

The second modification comes from the fact that with a non-zero source term, it is no longer true that $E_{, t}=-\rho u$. This means that the time interpolation described in $\$ 5.4 .2$ must be slightly modified. Instead of using $E_{, t}=-\rho u$, 84, and 85 , we make use of the following modified formulas:

$$
\begin{aligned}
E_{, t}(t, x) & =-\rho u+C_{1}, \\
E_{, t t}(t, x) & =\mathbb{E}_{, x}-\rho E+C_{2}, \\
E_{, t t t}(t, x) & =(2 \rho u E)_{, x}-\mathbb{F}_{, x x}+E(\rho u)_{, x}+\rho^{2} u+C_{3},
\end{aligned}
$$

where

$$
\begin{aligned}
& C_{1}:=\frac{\sqrt{\pi}}{4}+\frac{\sqrt{\pi}}{8}(4 \pi-1) \cos (2 x-2 \pi t), \\
& C_{2}:=\frac{3 \sqrt{\pi}+4 \pi-16 \sqrt{\pi^{5}}}{16} \sin (-2 x+2 \pi t)+\frac{\pi}{16} \sin (4 x-4 \pi t), \\
& C_{3}:=-\frac{\pi}{4}+\frac{7 \sqrt{\pi}+16 \pi-64 \sqrt{\pi^{7}}}{32} \cos (2 x-2 \pi t)-\frac{3 \pi}{16} \cos (4 x-4 \pi t) .
\end{aligned}
$$

In the above expression we used the shorthand notation:

$$
x:=x^{1}, \quad E:=E^{1}, \quad u:=u^{1}, \quad \mathbb{E}:=\mathbb{E}^{11}, \quad \text { and } \quad \mathbb{F}:=\mathbb{F}^{111} .
$$

We run the initial condition out to time $t=1$, at which point it should return to its initial state. Convergence studies on various grids on the domain $(x, v) \in[-\pi, \pi] \times[-\pi, \pi]$ with Strang and the fourth-order operator splitting results are shown in Table 3 . We compute the errors in an identical manner to the linear test problem presented in the previous section using the relative $L_{2}$ errors defined by equation (C.4) with $M=5$. See Appendix C for more details.

\subsection{Two-stream instability}

The two-stream instability problem has become a standard benchmark to test numerical Vlasov solvers, and has been used as such by several authors (e.g., 25, 2, 34, 22, 12, 9]). We use the following initial distribution function

$$
f(t=0, x, v)=\frac{v^{2}}{\sqrt{8 \pi}}\left(2-\cos \left(\frac{x}{2}\right)\right) e^{-\frac{v^{2}}{2}},
$$

and solve on the domain $(x, v) \in[-2 \pi, 2 \pi] \times[-2 \pi, 2 \pi]$. Results for time $t=45$ are presented in Figure 4 for various mesh sizes. In Figure 5 we present vertical cross-sections of the solution taken at $x=0$ for the same mesh sizes.

Figures 4 and 5 clearly demonstrate the ability of the discontinuous Galerkin methodology to approximate very rough data, something that is more difficult with methods that act over larger stencils. The results shown in these figures indicate far more structure than what is shown in other recent work, including results from the WENO method [34, 2] and an explicit DG method that uses a 


\begin{tabular}{|c||c|c||c|c|}
\hline Mesh & SL2 Error & $\log _{2}$ (Ratio) & SL4 Error & $\log _{2}$ (Ratio) \\
\hline \hline $10^{2}$ & $5.210 \times 10^{-1}$ & - & $9.493 \times 10^{-1}$ & - \\
\hline $20^{2}$ & $1.433 \times 10^{-1}$ & 1.86 & $2.715 \times 10^{-1}$ & 1.81 \\
\hline $40^{2}$ & $1.640 \times 10^{-2}$ & 3.13 & $1.652 \times 10^{-2}$ & 4.04 \\
\hline $80^{2}$ & $3.438 \times 10^{-3}$ & 2.26 & $7.079 \times 10^{-4}$ & 4.55 \\
\hline $160^{2}$ & $8.333 \times 10^{-4}$ & 2.04 & $3.434 \times 10^{-5}$ & 4.37 \\
\hline $320^{2}$ & $2.068 \times 10^{-4}$ & 2.01 & $1.962 \times 10^{-6}$ & 4.13 \\
\hline $640^{2}$ & $5.161 \times 10^{-5}$ & 2.00 & $1.203 \times 10^{-7}$ & 4.03 \\
\hline $1280^{2}$ & $1.290 \times 10^{-5}$ & 2.00 & $7.509 \times 10^{-9}$ & 4.00 \\
\hline
\end{tabular}

Table 3: Convergence study for the forced Vlasov-Poisson equation. All calculations presented here are $5^{\text {th }}$ order in space and were run with a CFL number of 5 . Shown are the relative errors computed via C.4 at time $t=1$. SL2 refers to the Strang split semi-Lagrangian scheme and SL4 to the fourth-order split semi-Lagrangian scheme. Since the positivity-preserving limiters as described in 5.6 don't guarantee positivity in the mean in the presence of a source term, we have turned them off for this convergence study only.

piecewise constant representation of the distribution function, $f$, and a piecewise quadratic representation of the electric potential $\phi[25$.

In Figure 6 we demonstrate the effects of adding the posivitity-preserving limiter. We see that even without limiting, the base scheme already does a reasonable job of not producing large negative values in the distribution function. With the positivity-preserving limiters we are able to remove these small positivity violations. In Figure 7 we plot four quantities that are exactly conserved by the continuous Vlasov-Poisson equation, but only approximately conserved in our numerical discretization: $L_{1}$-norm of $f(11), L_{2}$-norm of $f(12)$, total energy (13), and total entropy (14). In particular, we use the numerical approximations to (11)-14 as given by equations A.3 - A.6 in Appendix A We note that it is difficult to obtain accurate values for the total entropy (14), because there are many values where $f$ becomes very small.

\subsection{Weak Landau damping}

Landau damping has been extensively studied both numerically [9, 25, 45] and analytically (e.g., the work of Mouhot and Villani [32]). Just as the twostream instability problem, Landau damping has become a favorite standard test case. It is particularly useful since the linear decay rates of the $L_{2}$-norm of the electric field are well-known.

We use the following initial distribution function

$$
f(t=0, x, v)=\frac{1}{\sqrt{2 \pi}}(1+\alpha \cos (k x)) e^{-\frac{v^{2}}{2}},
$$



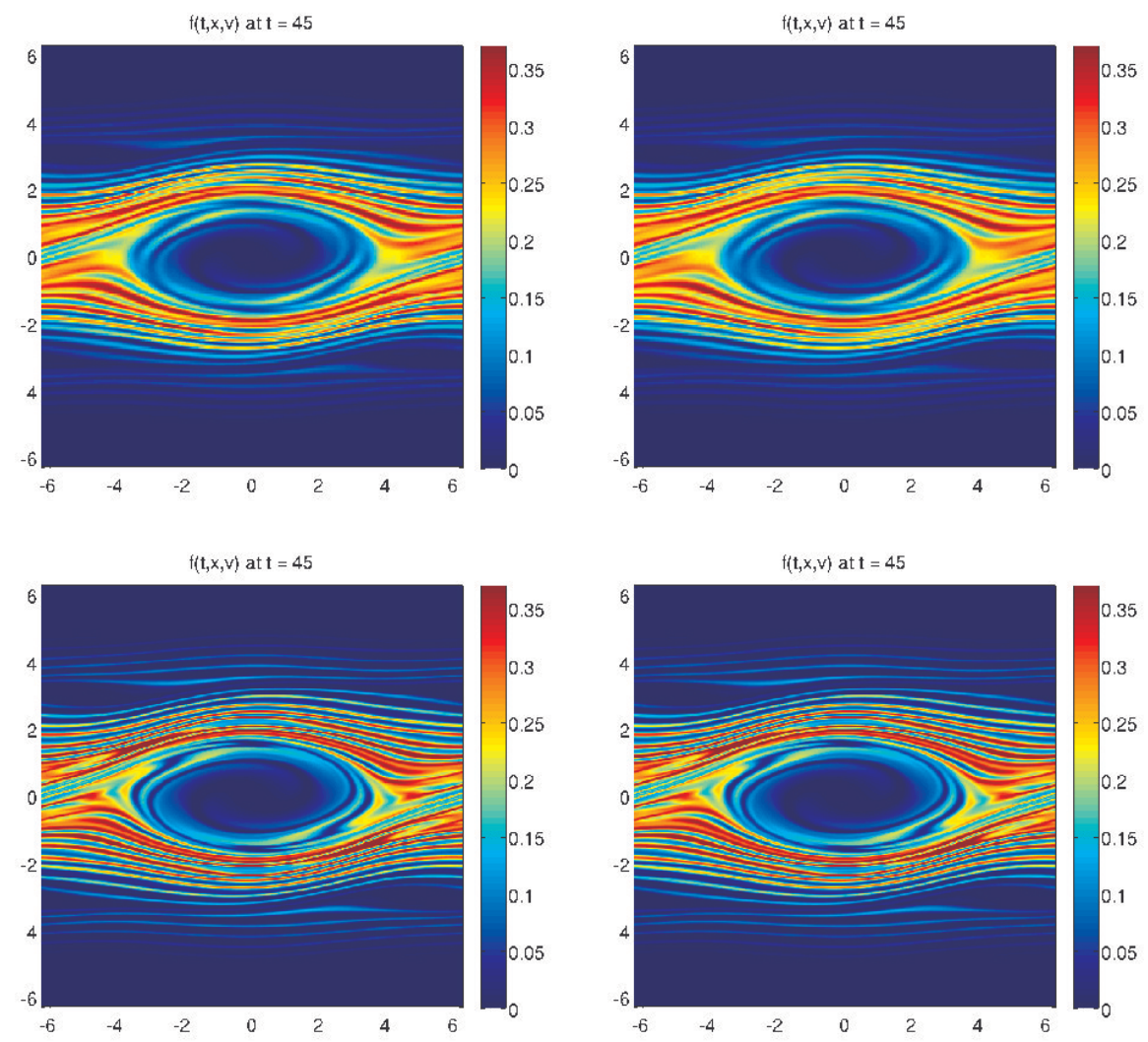

$f(t, x, v)$ at $t=45$

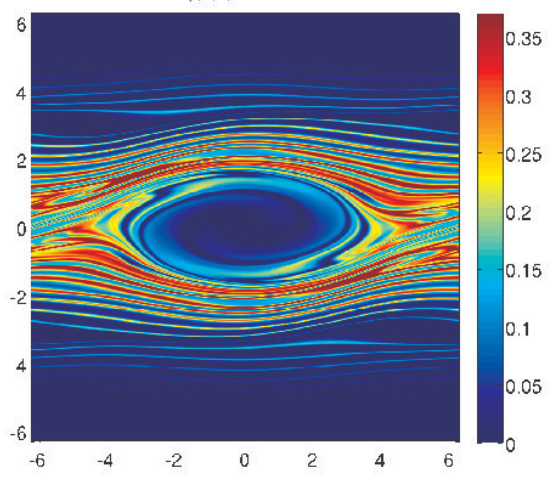

$f(t, x, v)$ at $t=45$

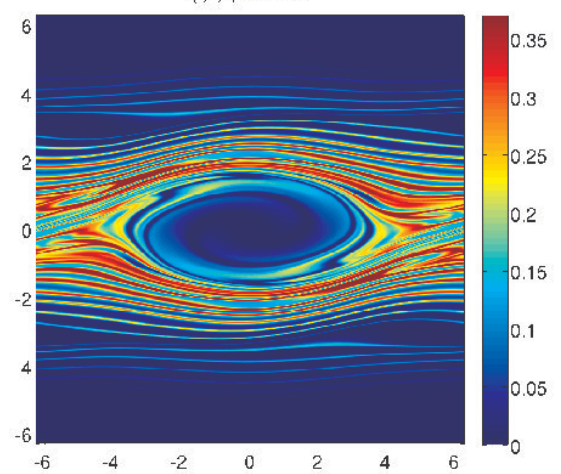

Figure 4: The two-stream instability problem. The panels in the left-hand column are results using the $2^{\text {nd }}$ order Strang splitting method. The panels in the right-hand column are results using the $4^{\text {th }}$ order splitting method. All simulations are $5^{\text {th }}$ order in space. The mesh sizes for the first, second and third rows are $\left(m_{x}, m_{v}\right)=(65,65),\left(m_{x}, m_{v}\right)=(129,129)$, and $\left(m_{x}, m_{v}\right)=(255,255)$, respectively. All solutions use the positivity-preserving algorithm. The above figures were produced by plotting the numerical solution at each of the $5 \times 5$ Gaussian quadrature points in each mesh element. 

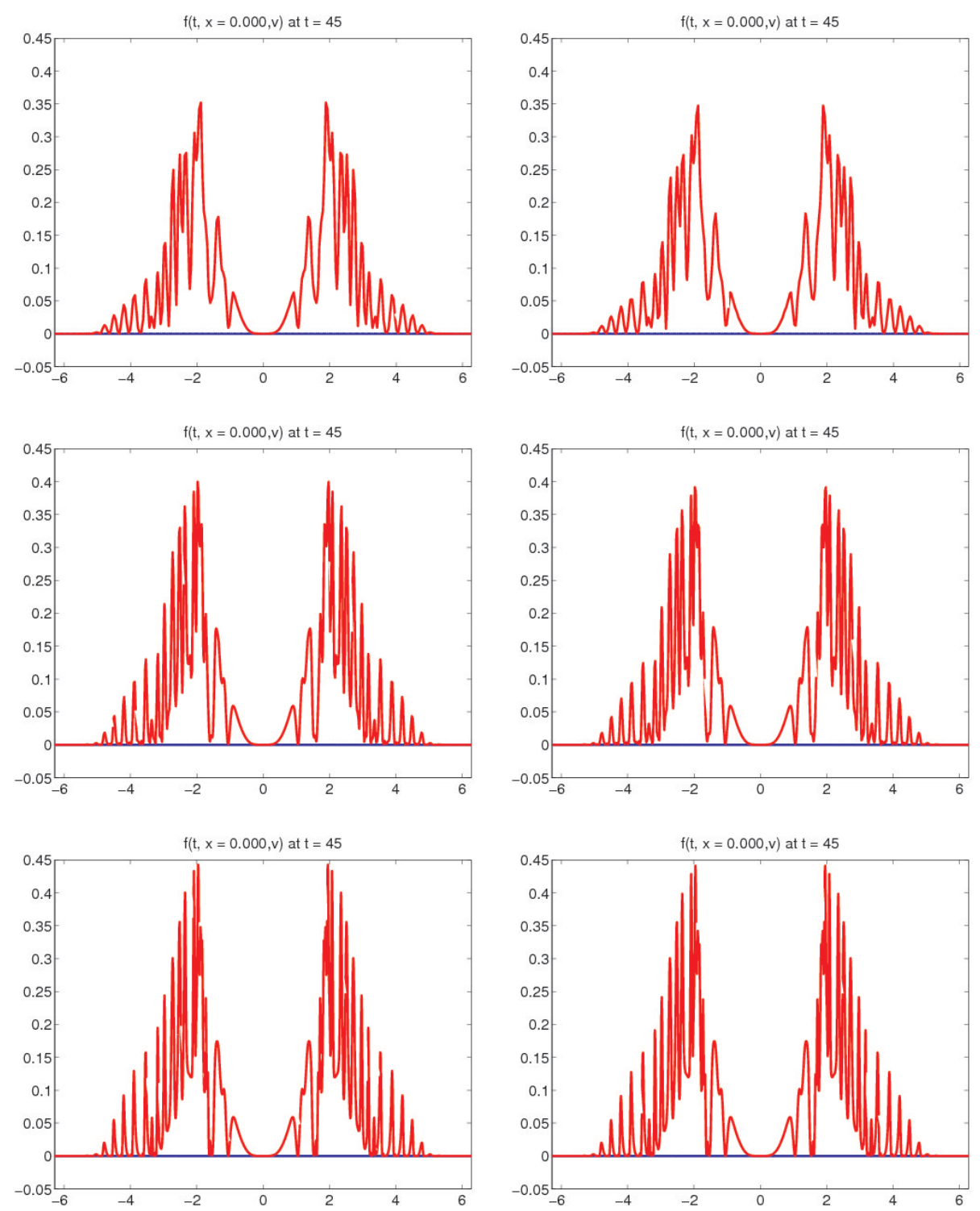

Figure 5: The two-stream instability problem. The panels in the left-hand column are results using the $2^{\text {nd }}$ order Strang splitting method. The panels in the right-hand column are results using the $4^{\text {th }}$ order splitting method. All simulations are $5^{\text {th }}$ order accurate in space. The mesh sizes for the first, second and third rows are $\left(m_{x}, m_{v}\right)=(65,65),\left(m_{x}, m_{v}\right)=(129,129)$, and $\left(m_{x}, m_{v}\right)=(255,255)$, respectively. All solutions use the positivity-preserving algorithm. Each figure above represents the numerical solution at $x=0$ and use 5 Gaussian quadrature points for each cell in the $v$-coordinate. The above solutions were computed with an odd number of elements in each coordinate direction in order to easily obtain a slice of the solution at $x=0$. 

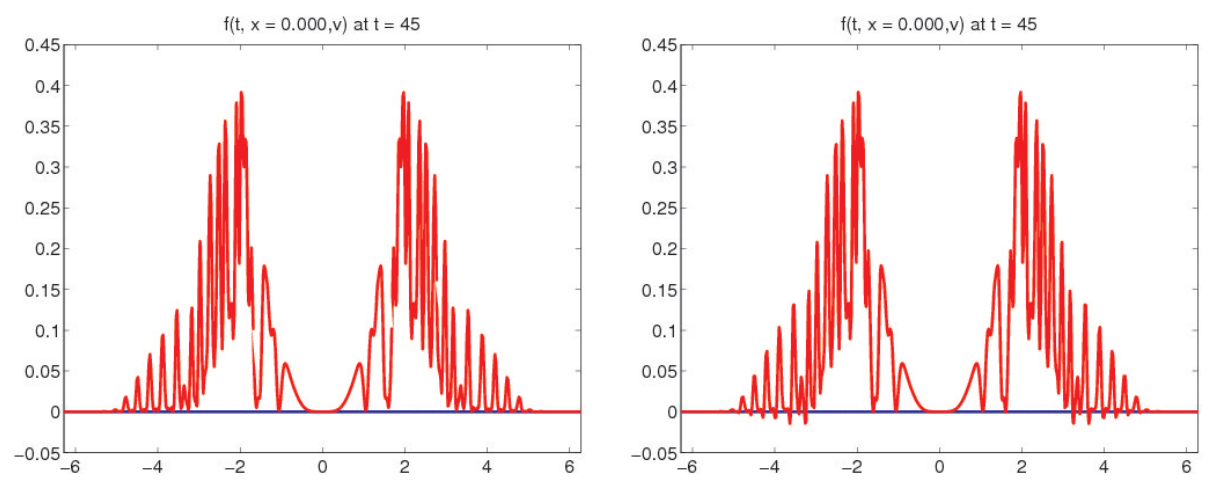

Figure 6: The two-stream instability problem. These panels demonstrate the effect of the positivity preserving-limiter. The result on the left is the $4^{\text {th }}$ order splitting algorithm with limiters turned on, and the result on the right hand side is the same algorithm with the limiters turned off. Both pictures represent a slice of the solution at $x=0$ and final time $t=45$. Both results represent a mesh of size $\left(m_{x}, m_{v}\right)=(129,129)$ and are $5^{\text {th }}$ order accurate in space; an odd number of grid elements are used in order to easily obtain function values. We further note that $\min _{i} f\left(x_{i}, v_{i}\right)=-2.020 \times 10^{-2}$ for the solution without the limiter and $\min _{i} f\left(x_{i}, v_{i}\right)=7.000 \times 10^{-12}$ for the limited solution, where the minimum is taken over all $5 \times 5$ Gaussian quadrature points over every mesh element.

with $\alpha=0.01$ and $k=0.5$ on the domain $(x, v) \in[-2 \pi, 2 \pi] \times[-2 \pi, 2 \pi]$. Because $\alpha$ is a small parameter, we expect to see results that closely agree with the linear theory, where the electric field decays exponentially. In Figure 8 we present this decay provided by

$$
\log \left(\|E(t, \cdot)\|_{L_{2}}\right):=\log \sqrt{\int_{-2 \pi}^{2 \pi}|E(t, x)|^{2} d x}
$$

versus time for two different mesh sizes. Our computed decay rate matches the linear decay rate, $\gamma=-0.1533$. In Figure 9 we again plot the deviations of several quantities that are conserved by the continuous Vlasov-Poisson system from their initial values: $\|f\|_{L_{1}},\|f\|_{L_{2}}$, total energy, and entropy. We again use the numerical approximation to these norms given by A.3 - A.6) in Appendix A Our results are comparable to what is reported for example by Qiu and Christlieb 34].

\subsection{Strong Landau damping}

The initial condition is again given by (125), this time with $\alpha=0.5$ and $k=0.5$ on the domain $(x, v) \in[-2 \pi, 2 \pi] \times[-2 \pi, 2 \pi]$. The time evolution of the distribution function is shown in the panels of Figure 10 . These images are comparable to what is shown in Qiu and Christlieb [34, but we are again able to capture more fine scale structure with the discontinuous Galerkin approach.

A semi-log plot of the $L_{2}$-norm of the electric field is provided in Figure 11. and decay rates are computed by sampling the solution at data points. 

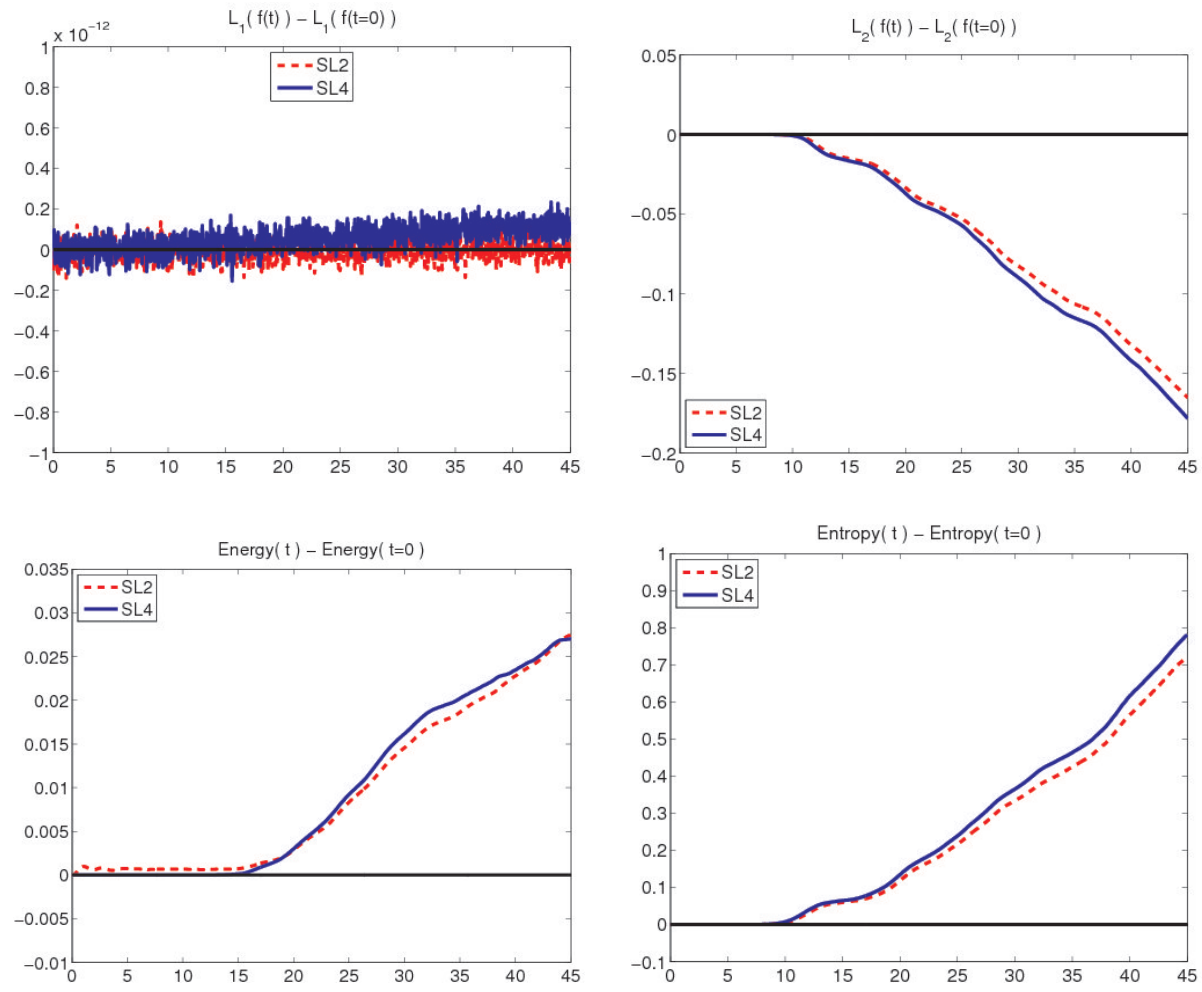

Figure 7: The two-stream instability problem. Shown in these panels are the deviations of the $L_{1}$ norm of $f$ (top-left), $L_{2}$ norm of $f$ (top-right), total energy (bottom-left), and total entropy (bottom-right) from their initial values. All simulations use a constant CFL number of 2.0, and are obtained from a mesh of size $\left(m_{x}, m_{v}\right)=(129,129)$. The domain for this problem is $(x, v) \in[-2 \pi, 2 \pi] \times[-2 \pi, 2 \pi]$. Each simulation is $5^{\text {th }}$ order accurate in space and is positivity preserving. 

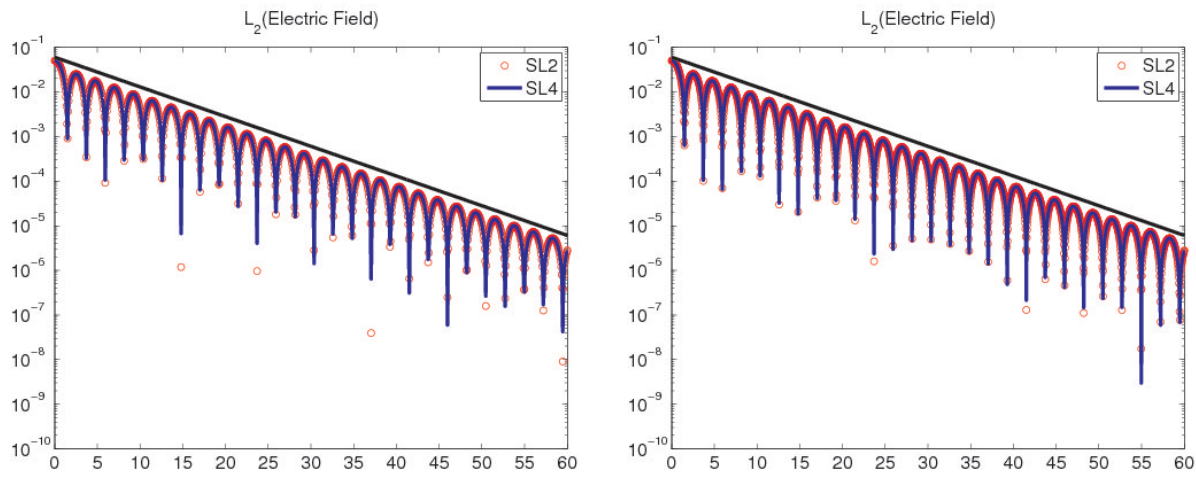

Figure 8: The weak Landau damping problem. Shown in the panels are semi-log plots of the $L_{2}$-norm of the electric field. Simulations use a constant CFL number of 2.0 and are $5^{\text {th }}$ order accurate in space. All simulations use the positivity-preserving limiter. The figure on the left represents a mesh of size $\left(m_{x}, m_{v}\right)=(64,128)$ and the result on the right was represents a mesh of size $\left(m_{x}, m_{v}\right)=(128,256)$, both on the domain $(x, v) \in[-2 \pi, 2 \pi] \times[-2 \pi, 2 \pi]$. Both simulations match the theoretical decay rate of $\gamma=-0.1533$, and to demonstrate this we plot the line defined by $y=0.06 e^{\gamma t}$. One should note that the discrepancy in the two plots is due to the fact that twice as many time points in the second plot as the first one.

We find that the initial linear decay rate is approximately $\gamma_{1} \approx-0.292$ which is close to the value of -0.243 computed by Zaki et al. [43], closer still to the value of -0.281 computed by Cheng and Knorr 9, but much larger than the value of -0.126 computed by Heath et. al 25]. In this same figure we also estimate the growth rate due to particle trapping and find it to be approximately $\gamma_{2}=0.0815$; this number also differs from the value reported by Heath et al. 25]: $\gamma_{2}=0.0324$. The initial linear decay was computed by taking the maximum of the first two peaks located at $t \approx 2.45$ and $t \approx 4.54$. For the particle trapping growth regime, we sampled the maximum of the solution at the two peaks located at $t \approx 2.33$ and $t \approx 2.84$. We postulate that the difference between our computed growth rates and those of Heath et al. 25] stems from the fact that we are using piecewise quartic polynomials to represent the distribution function, while they are using only piecewise constants. This issue should be further investigated.

In Figure 12 we again plot the deviations of several quantities that are conserved by the continuous Vlasov-Poisson system from their initial values: $\|f\|_{L_{1}},\|f\|_{L_{2}}$, total energy, and entropy. In particular, we use the numerical approximations to (11)- (14) as given by equations (A.3)-A.6) in Appendix A. Our results are comparable to what is reported for example by Qiu and Christlieb [34.

Finally, we note that in both our weak and strong Landau damping simulations we made $V_{\max }=2 \pi$ instead of the more commonly used value of $V_{\max }=5$. The reason for this is that we noticed that between roughly $v=5$ and $v=6$ the distribution function $f(t, x, v)$ still had a non-negligable amplitude on the 

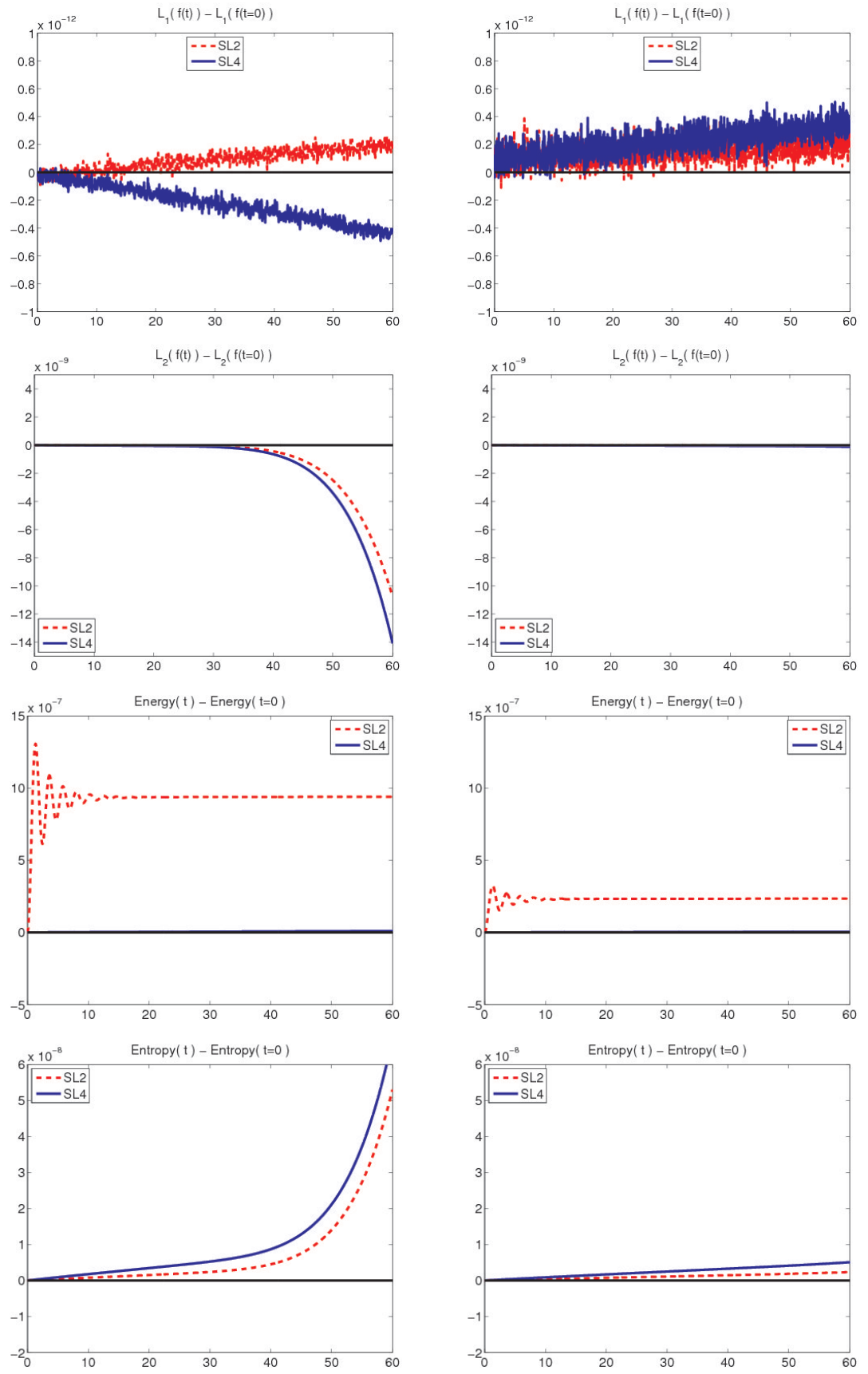

Figure 9: The weak Landau damping problem. Shown in the panels are the deviations of various quantities that are conserved by the exact equations from their initial values. All simulations use a constant CFL number of 2.0 , are $5^{\text {th }}$ order accurace in space, and are positivity preserving. The mesh size for the left hand column is $\left(m_{x}, m_{v}\right)=(64,128)$ and the mesh size for the right hand column is $\left(m_{x}, m_{v}\right)=(128,256)$. We note that the largest deviation for total energy for the $4^{\text {th }}$ order algorithm is on the order of $10^{-10}$. 
order of about $10^{-6}$; the precise behavior of strong and weak Landau damping in this region is shown in Figure 13 Therefore, truncating at $V_{\max }=5$ caused additional errors when tracking various conserved quantities; we found improvements in these errors when taking $V_{\max }=2 \pi$.

\section{Conclusions and future work}

We have described in this work a semi-Lagrangian discontinuous Galerkin method for solving the $1+1$ Vlasov-Poisson system. This method was shown to have all of the following properties:

1. Unconditionally stable;

2. High-order accurate in space ( $5^{\text {th }}$ order);

3. High-order accurate in time ( ${ }^{\text {th }}$ order);

4. Mass conservative; and

5. Positivity-preserving.

The proposed method is based on a series quasi-1D semi-Lagrangian advection steps coupled with a fourth-order operator splitting scheme. The spatial discretization is handled via high-order discontinuous Galerkin representations. The Poisson equation is solved to high-order via a modified local DG method, where the boundary conditions are set so that the discrete Laplacian matrix is by construction LU factored. The scheme was applied to several standard Vlasov-Poisson test cases, which demonstrated the accuracy and robustness of the proposed scheme.

The advantage of DG over other methods that are based on larger stencils is its ability to represent very rough data. We showed that this feature is important in the case of the two-stream instability and the Landau damping calculations presented in $\$ 6$. With an explicit time-stepping method, the price that is paid for this spatial localization is a maximum CFL number that decreases with the spatial order of accuracy. In the context of Vlasov-Poisson we have tamed this problem by using the semi-Lagrangian framework.

Future work will focus on extending the results described in this paper to higher-dimensional Vlasov-Poisson equations. Furthermore, modifications of the current approach to both the non-relativisitc and the relativistic Vlasov-Maxwell equations will be considered.

Acknowledgements. The authors would like to thank the anonymous reviewers for their valuable feedback. This work was supported in part by NSF grants DMS-0711885 and DMS-1016202.

\section{Appendix A. Numerical evaluation of conserved quantities}

The conserved quantities defined in (11) $\left(L_{1}\right.$-norm), $12 p\left(L_{2}\right.$-norm), 13 ) (total energy), and (14) (entropy) are used as diagnostics of the numerical methods proposed in this work. 

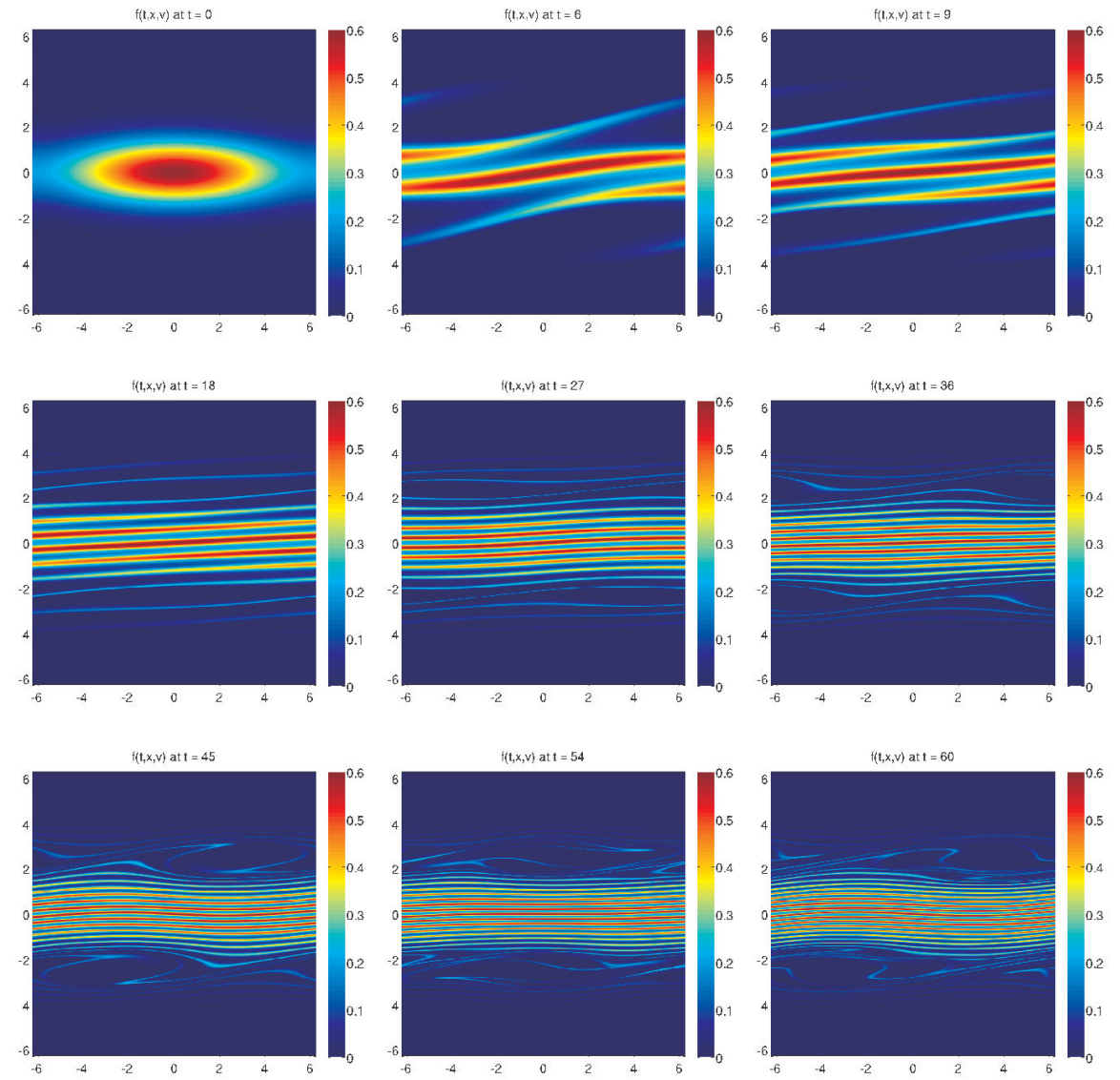

Figure 10: The strong Landau damping problem. Shown in the panels are the distribution function at various points in time. This simulation was run with a constant CFL number of 2.0 on a mesh of size $\left(m_{x}, m_{v}\right)=(128,256)$ using $5^{\text {th }}$ order accuracy in space and the positivity-preserving limiters. It is clear from these plots that the high-order discontinuous Galerkin method is able to capture much of the fine-scale structure for the solution. 

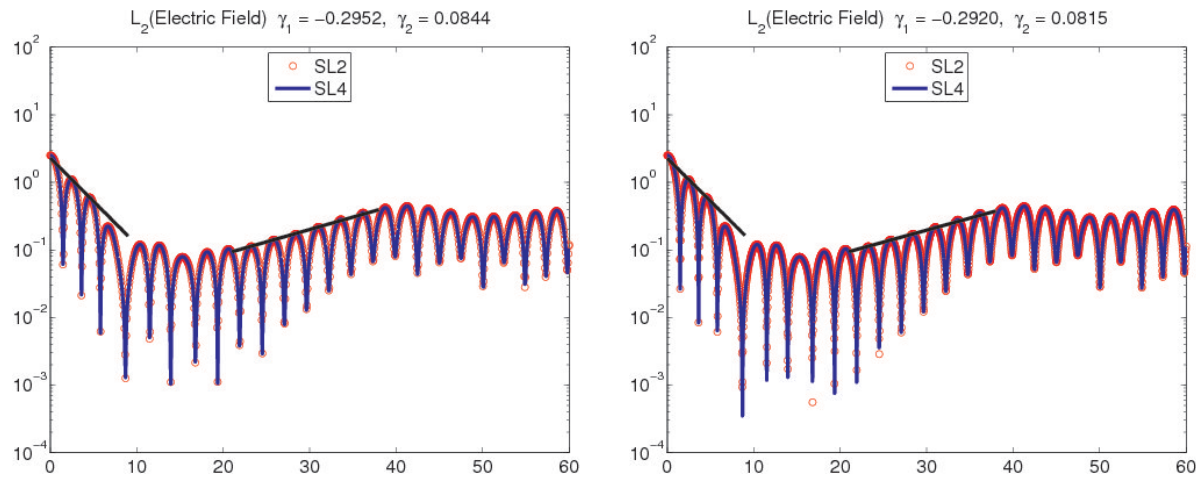

Figure 11: The strong Landau damping problem. Shown in these panels are semi-log plots of the $L_{2}$ norm of the electric field with two different resolutions; the mesh size for the the figure on the left is $\left(m_{x}, m_{v}\right)=(64,128)$ and the figure on the right is $\left(m_{x}, m_{v}\right)=(128,256)$. Both simulations use the positivity preserving limiter, are $5^{\text {th }}$ order accuracy in space and use a constant CFL number of 2.0. In each panel, $\gamma_{1}$ refers to the slope of the initial decay, and $\gamma_{2}$ refers to the growth rate between times $t=20$ and $t=40$.

In order to evaluate all of these conserved quantities in the numerical evolution, we define the following functional:

$$
I^{h}\left(g\left(f^{h}\right)\right):=\frac{\Delta x \Delta v}{4} \sum_{i=1}^{m_{x}} \sum_{j=1}^{m_{v}} \sum_{k=1}^{M^{2}} \omega_{k} g\left(f^{h}\left(x_{i}+\frac{\xi_{k} \Delta x}{2}, v_{j}+\frac{\eta_{k} \Delta v}{2}\right)\right),
$$

where $m_{x}$ is the number of elements in the $x$-direction, $m_{v}$ is the number of elements in the $v$-direction, and $\omega_{k}$ and $\left(\xi_{k}, \eta_{k}\right)$ are the $M^{2}$ Gauss-Legendre quadrature weights and points on $[-1,1] \times[-1,1]$, respectively. Expression (A.1) gives a numerical approximation to integrals of the form:

$$
I(g(f)):=\int_{-L}^{L} \int_{-\infty}^{\infty} g(f(x, v)) d v d x .
$$

Using A.1 we define the following numerical approximations to the norms defined by (11)-(14):

$$
\begin{aligned}
\left\|f^{h}\right\|_{L_{1}} & :=I^{h}\left(\left|f^{h}\right|\right), \\
\left\|f^{h}\right\|_{L_{2}} & :=\left\{\frac{\Delta x \Delta v}{4} \sum_{i=1}^{m_{x}} \sum_{j=1}^{m_{v}} \sum_{\ell=1}^{M(M+1) / 2}\left[F_{i j}^{(\ell)}\right]^{2}\right\}^{\frac{1}{2}}, \\
\text { Total energy } & :=\frac{1}{2} I^{h}\left(v^{2} f^{h}\right)+\frac{\Delta x}{4} \sum_{i=1}^{m_{x}} \sum_{\ell=1}^{M}\left[E_{i}^{(\ell)}\right]^{2}, \\
\text { Entropy } & :=-I^{h}\left(f^{h} \log \left(f^{h}\right)\right) .
\end{aligned}
$$



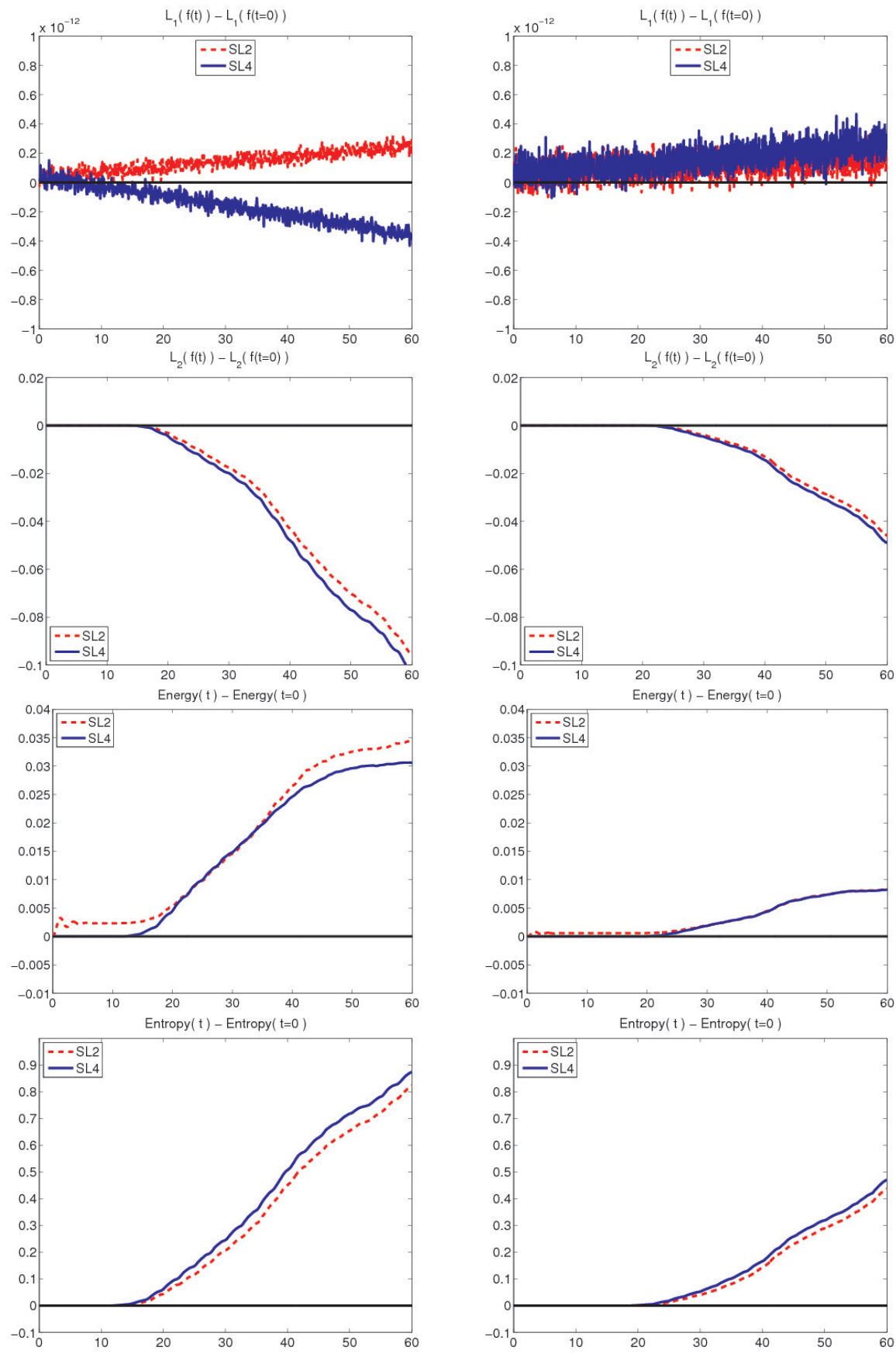

Figure 12: The strong Landau damping problem. Simulation results for the $L_{1}$ norm (first row), $L_{2}$ norm (second row), energy (third row), and entropy (bottom row) for strong Landau damping. All simulations use a constant CFL number of 2.0 and are $5^{\text {th }}$ order accurate in space. The mesh size for the left column is $\left(m_{x}, m_{v}\right)=(64,128)$ and the mesh size for the right column is $\left(m_{x}, m_{v}\right)=(128,256)$. 

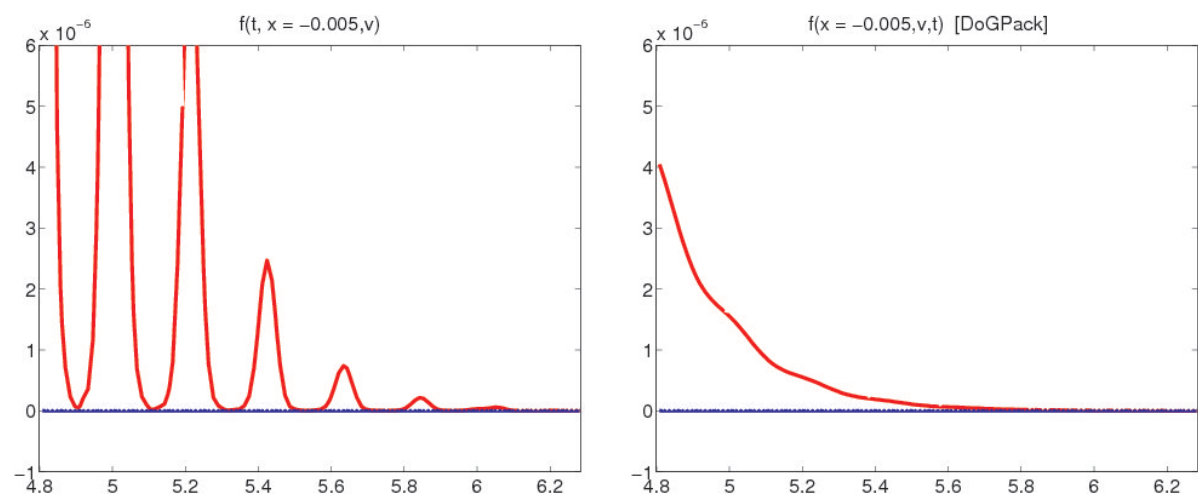

Figure 13: A comparison of vertical slices for strong landau damping (left) and weak Landau damping (right) at time $t=60$. The simulations for each panel use a grid resolution of $\left(m_{x}, m_{v}\right)=(128,256)$, each are $5^{\text {th }}$ order accuracy in space, and each use the positivitypreserving limiter. The CFL number for each simulation is 2.0. We note that the solution is non-zero for $|v|>5$ in both cases, although there is much more activity in the case of strong Landau damping. The plots suggests that the commonly used maximum velocity of $|v|=5$ should be increased in order to get better accuracy in conservation of the quantities A.3 - A.6.

\section{Appendix B. Relative $L_{2}$-norm error in $1 \mathrm{D}$}

Let $f(x)$ be the exact solution of some problem of interest. Let $f^{h}(x)$ denote an approximation to $f(x)$ using a discontinuous Galerkin method. On each element $f^{h}(x)$ and $f(x)$ can be written as

$$
\begin{aligned}
\left.f^{h}(x)\right|_{\mathcal{T}_{i}} & =\sum_{k=1}^{M} F_{i}^{(k)} \varphi^{(k)}(\xi), \\
\left.f(x)\right|_{\mathcal{T}_{i}} & =\sum_{k=1}^{\infty} \mathcal{F}_{i}^{(k)} \varphi^{(k)}(\xi),
\end{aligned}
$$

respectively. The relative $L_{2}$-norm of the difference on the domain $x \in[a, b]$ between the approximation, $f^{h}(x)$, and the exact solution, $f(x)$, is given by

$$
\begin{aligned}
\frac{\left\|f(x)-f^{h}(x)\right\|_{L_{2}}}{\|f(x)\|_{L_{2}}} & =\left\{\frac{\int_{a}^{b}\left[f(x)-f^{h}(x)\right]^{2} d x}{\int_{a}^{b} f(x)^{2} d x}\right\}^{\frac{1}{2}} \\
& =\left\{\frac{\sum_{i=1}^{N} \sum_{k=1}^{M}\left[F_{i}^{(k)}-\mathcal{F}_{i}^{(k)}\right]^{2}}{\sum_{i=1}^{N} \sum_{k=1}^{M}\left[\mathcal{F}_{i}^{(k)}\right]^{2}}\right\}^{\frac{1}{2}}+\mathcal{O}\left(\Delta x^{M}\right),
\end{aligned}
$$


where $N$ is the total number of grid elements and $\Delta x=(b-a) / N$. Therefore, we take as our relative $L_{2}$-norm indicator the following easily computable quantity:

$$
E_{2}(\Delta x, M):=\left\{\frac{\sum_{i=1}^{N} \sum_{k=1}^{M}\left[F_{i}^{(k)}-\mathcal{F}_{i}^{(k)}\right]^{2}}{\sum_{i=1}^{N} \sum_{k=1}^{M}\left[\mathcal{F}_{i}^{(k)}\right]^{2}}\right\}^{\frac{1}{2}} .
$$

\section{Appendix C. Relative $L_{2}$-norm error in $2 \mathrm{D}$}

Let $f(x, y)$ be the exact solution of some problem of interest. Let $f^{h}(x, y)$ denote an approximation to $f(x, y)$ using a discontinuous Galerkin method. On each element $f^{h}(x, y)$ and $f(x, y)$ can be written as

$$
\begin{aligned}
\left.f^{h}(x, y)\right|_{\mathcal{T}_{i j}} & =\sum_{k=1}^{M(M+1) / 2} F_{i j}^{(k)} \varphi^{(k)}(\xi, \eta), \\
\left.f(x, y)\right|_{\mathcal{T}_{i j}} & =\sum_{k=1}^{\infty} \mathcal{F}_{i j}^{(k)} \varphi^{(k)}(\xi, \eta),
\end{aligned}
$$

respectively. The relative $L_{2}$-norm of the difference on the domain $(x, y) \in$ $\left[a_{x}, b_{x}\right] \times\left[a_{y}, b_{y}\right]$ between the approximation, $f^{h}(x, y)$, and the exact solution, $f(x, y)$, is given by

$$
\begin{aligned}
& \frac{\left\|f(x, y)-f^{h}(x, y)\right\|_{L_{2}}}{\|f(x, y)\|_{L_{2}}}=\left\{\frac{\int_{a_{x}}^{b_{x}} \int_{a_{y}}^{b_{y}}\left[f(x, y)-f^{h}(x, y)\right]^{2} d y d x}{\int_{a_{x}}^{b_{x}} \int_{a_{y}}^{b_{y}} f(x, y)^{2} d y d x}\right\}^{\frac{1}{2}} \\
& =\left\{\frac{\sum_{i=1}^{N_{x}} \sum_{j=1}^{N_{y}} \sum_{k=1}^{M(M+1) / 2}\left[F_{i j}^{(k)}-\mathcal{F}_{i j}^{(k)}\right]^{2}}{\sum_{i=1}^{N_{x}} \sum_{j=1}^{N_{y}} \sum_{k=1}^{M(M+1) / 2}\left[\mathcal{F}_{i j}^{(k)}\right]^{2}}\right\}^{\frac{1}{2}}+\mathcal{O}\left(\Delta x^{M}, \Delta y^{M}\right),
\end{aligned}
$$

where $N_{x}$ and $N_{y}$ are the the number of grid elements in each coordinate direction, $\Delta x=\left(b_{x}-a_{x}\right) / N_{x}$, and $\Delta y=\left(b_{y}-a_{y}\right) / N_{y}$. Therefore, we take as our relative $L_{2}$-norm indicator the following easily computable quantity:

$$
E_{2}(\Delta x, \Delta y, M):=\left\{\frac{\sum_{i=1}^{N_{x}} \sum_{j=1}^{N_{y}} \sum_{k=1}^{M(M+1) / 2}\left[F_{i j}^{(k)}-\mathcal{F}_{i j}^{(k)}\right]^{2}}{\sum_{i=1}^{N_{x}} \sum_{j=1}^{N_{y}} \sum_{k=1}^{M(M+1) / 2}\left[\mathcal{F}_{i j}^{(k)}\right]^{2}}\right\}^{\frac{1}{2}} .
$$

\section{References}

[1] D.N. Arnold, F. Brezzi, B. Cockburn, and L.D. Marini. Unified analysis of discontinuous Galerkin methods for elliptic problems. SIAM J. Numer. Anal., 39:1749-1779, 2002. 
[2] J.W. Banks and J.A.F. Hittinger. A new class of nonlinear finite-volume methods for Vlasov simulation. IEEE Transactions on Plasma Science, $38: 2198-2207,2010$.

[3] J. Barnes and P. Hut. A hierarchical $\mathrm{O}(N \log N)$ force-calculation algorithm. Nature, 324:446 - 449, 1986.

[4] R. Belaouar, N. Crouseilles, P. Degond, and E. Sonnendrücker. An asymptotically stable semi-Lagrangian scheme in the quasi-neutral limit. J. Sci. Comput., 41:341-365, 2009.

[5] N. Besse, J. Segre, and E. Sonnendrücker. Semi-Lagrangian schemes for the two-dimensional Vlasov-Poisson system on unstructured meshes. Transp. Theory and Stat. Phys., 34:311-332, 2005.

[6] N. Bessho and A. Bhattacharjee. Fast collisionless reconnection in electronpositron plasmas. Physics of Plasmas, 14:056503, 2007.

[7] C.K. Birdsall and A.B. Langdon. Plasma physics via computer simulation. Taylor \& Francis Group, 1985.

[8] J. Birn, J.F. Drake, M.A. Shay, B.N. Rogers, R.E. Denton, M. Hesse, M. Kuznetsova, Z.W. Ma, A. Bhattacharjee, A. Otto, and P.L. Pritchett. Geospace environmental modeling (GEM) magnetic reconnection challenge. Journal of Geophysical Research - Space Physics, 106(A3):3715-3719, 2001.

[9] C. Cheng and G. Knorr. The integration of the Vlasov equation in configuration space. J. Comp. Phys., 22:330-351, 1976.

[10] A.J. Christlieb, W.N.G. Hitchon, and E.R. Keiter. A computational investigation of the effects of varying discharge geometry for an inductively coupled plasma. IEEE Transactions on Plasma Science, 28:2214-2231, 2000.

[11] A.J. Christlieb, R. Krasny, and J.P. Verboncoeur. Efficient particle simulation of a virtual cathode using a grid-free treecode poisson solver. IEEE Transactions on Plasma Science, 32:384-389, 2004.

[12] A.J. Christlieb, R. Krasny, J.P. Verboncoeur, J.W. Emhoff, and I.D. Boyd. Grid-free plasma simulation techniques. IEEE Transactions on Plasma Science, 34:149-165, 2006.

[13] B. Cockburn, S. Hou, and C.-W. Shu. TVB Runge-Kutta local projection discontinuous Galerkin finite element method for conservation laws IV: The multidimensional case. Math. Comp., 54:545, 1990.

[14] B. Cockburn, S.Y. Lin, and C.-W. Shu. TVB Runge-Kutta local projection discontinuous Galerkin finite element method for conservation laws III: One dimensional systems. J. Comp. Phys., 84:90, 1989. 
[15] B. Cockburn and C.-W. Shu. TVB Runge-Kutta local projection discontinuous Galerkin finite element method for scalar conservation laws II: General framework. Math. Comp., 52:411-435, 1989.

[16] B. Cockburn and C.-W. Shu. The Runge-Kutta local projection $P^{1}$ discontinuous Galerkin method for scalar conservation laws. $M^{2} A N, 25: 337$, 1991.

[17] B. Cockburn and C.-W. Shu. The Runge-Kutta discontinuous Galerkin method for conservation laws V: Multidimensional systems. J. Comp. Phys., 141:199-224, 1998.

[18] O. Coulaud, E. Sonnendrücker, E. Dillon, P. Bertrand, and A. Ghizzo. Parallelization of semi-Lagrangian Vlasov codes. J. Plasma Phys., 61:435448, 1999.

[19] N. Crouseilles, G. Latu, and E. Sonnendrücker. Hermite spline interpolation on patches for parallelly solving the Vlasov-Poisson equation. Int. J. Appl. Math. and Comp. Sci., 17:335-349, 2007.

[20] N. Crouseilles, M. Mehrenberger, and E. Sonnendrücker. Conservative semi-Lagrangian schemes for Vlasov equations. J. Comp. Phys., 229:19271953, 2010.

[21] N. Crouseilles, T. Respaud, and E. Sonnendrücker. A forward semiLagrangian method for the numerical solution of the Vlasov equation. Comp. Phys. Comm., 180:1730-1745, 2009.

[22] F. Filbet and E. Sonnendrücker. Comparison of Eulerian Vlasov solvers. Comp. Phys. Comm., 150:247-266, 2003.

[23] E. Forest and R.D. Ruth. Fourth-order symplectic integration. Physica D: Nonlinear Phenomena, 43:105-117, 1990.

[24] L. Greengard and V. Rokhlin. A fast algorithm for particle simulations. J. Comp. Phys., 73:325-348, 1987.

[25] R.E. Heath, I.M. Gamba, P.J. Morrison, and C. Michler. A discontinuous Galerkin method for the Vlasov-Poisson system. http://arxiv.org/abs/1009.3046v1, 2010.

[26] J.S. Hesthaven and T. Warburton. Nodal Discontinuous Galerkin Methods: Algorithms, Analysis, and Applications. Springer, 2007.

[27] R.W. Hockney and J.W. Eastwood. Computer simulation using particles. Institute of Physics Publishing, 1988.

[28] Y. Idomura, M. Ida, and S. Tokuda. Conservative gyrokinetic Vlasov simulation. Communications in Nonlinear Science and Numerical Simulation, 13:227-233, 2008. 
[29] G.B. Jacobs and J.S. Hesthaven. High-order nodal discontinuous Galerkin particle-in-cell method on unstructured grids. J. Comp. Phys., 96-121(214), 2006.

[30] G.B. Jacobs and J.S. Hesthaven. Implicit-explicit time integration of a highorder particle-in-cell method with hyperbolic divergence cleaning. Comp. Phys. Comm., 180:1760-1767, 2009.

[31] K. Lindsay and R. Krasny. A particle method and adaptive treecode for vortex sheet motion in three-dimensional flow. J. Comp. Phys., 172:879907, 2001.

[32] C. Mouhot and C. Villani. Landau damping. J. Math. Phys., 51(015204), 2010 .

[33] G.J. Parker and W.N.G. Hitchon. Convected scheme simulations of the electron distribution function in a positive column plasma. Jpn. J. Appl. Phys., 36:4799-4807, 1997.

[34] J. Qiu and A.J. Christlieb. A conservative high order semi-Lagrangian method for the Vlasov equation. J. Comp. Phys., 2010. Article in press.

[35] J. Qiu, M. Dumbser, and C.-W. Shu. The discontinuous Galerkin method with Lax-Wendroff type time discretizations. Comput. Methods Appl. Mech. Engr., 194:4528-4543, 2005.

[36] M. Restelli, L. Bonaventura, and R. Sacco. A semi-Lagrangian discontinuous Galerkin method for scalar advection by incompressible flows. J. Comp. Phys., 216:195-215, 2006.

[37] H. Schmitz and R. Grauer. Darwin-Vlasov simulations of magnetised plasmas. J. Comp. Phys., 214:738-756, 2006.

[38] E. Sonnendrücker, J. Roche, P. Bertrand, and A. Ghizzo. The semiLagrangian method for the numerical resolution of the Vlasov equation. J. Comp. Phys., 149:201-220, 1999.

[39] G. Strang. On the construction and comparison of difference schemes. SIAM J. Num. Anal., pages 506-517, 1968.

[40] J.-L. Vay, P. Colella, J.W. Kwan, P. McCorquodale, D.B. Serafini, A. Friedman, D.P. Grote, G. Westenskow, J.-C. Adam, A. Héron, and I. Haber. Application of adaptive mesh refinement to particle-in-cell simulations of plasmas and beams. Phys. Plasmas, 11(2928), 2004.

[41] H. Yoshida. Construction of higher order symplectic integrators. Phys. Lett. A, 150:262-268, 1990.

[42] H. Yoshida. Recent progress in the theory and application of symplectic integrators. Celest. Mech. and Dyn. Astro., 56:27-43, 1993. 
[43] S.I. Zaki, L.R.T. Gardner, and T.J.M. Boyd. A finite element code for the simulation of one-dimensional Vlasov plasmas. 2. Applications. J. Comp. Phys., 79:200-208, 1988.

[44] X. Zhang and C.-W. Shu. On maximum-principle-satisfying high order schemes for scalar conservation laws. J. Comp. Phys., 229:3091-3120, 2010.

[45] T. Zhou, Y. Guo, and C.W. Shu CW. Numerical study on landau damping. Physica D: Nonlinear Phenomena, 157:322-333, 2001. 